\title{
Dynamical exchange-correlation potentials for an electron liquid
}

\author{
Zhixin Qian and Giovanni Vignale \\ Department of Physics, University of Missouri, Columbia, Missouri 65211
}

(Received 22 January 2002; published 18 June 2002)

\begin{abstract}
The imaginary parts of the exchange-correlation kernels $f_{\mathrm{xc}}^{L, T}(\omega)$ in the longitudinal and transverse currentcurrent response functions of a homogeneous electron liquid are calculated exactly at low frequency, to leading order in the Coulomb interaction. Combining these new results with the previously known high-frequency behaviors of $\operatorname{Im} f_{\mathrm{xc}}^{L, T}(\omega)$ and with the compressibility and the third moment sum rules, we construct simple interpolation formulas for $\operatorname{Im} f_{\mathrm{xc}}^{L, T}(\omega)$ in three and two spatial dimensions. A feature of our interpolation formulas is that they explicitly take into account the two-plasmon component of the excitation spectrum: our longitudinal spectrum $\operatorname{Im} f_{\mathrm{xc}}^{L}(\omega)$ is thus intermediate between the Gross-Kohn interpolation, which ignores the two-plasmon contribution, and a recent approximate calculation by Nifosì, Conti, and Tosi, which probably overestimates it. Numerical results for both the real and imaginary parts of the exchange-correlation kernels at typical electron densities are presented, and compared with those obtained from previous approximations. We also find an exact relation between $\operatorname{Im} f_{\mathrm{xc}}^{L}(\omega)$ and $\operatorname{Im} f_{\mathrm{xc}}^{T}(\omega)$ at small $\omega$.
\end{abstract}

DOI: 10.1103/PhysRevB.65.235121

PACS number(s): 71.15.Mb, 71.10.- w, 71.45.Gm

\section{INTRODUCTION}

Just as the ground-state density functional theory ${ }^{1,2}$ maps a real interacting many-electron system to an effective noninteracting one with the same density, the time-dependent density functional theory (TDDFT) ${ }^{3,4}$ maps a time-dependent many-body system subjected to an external potential $v(\mathbf{r}, t)$ to a noninteracting system with the same density $n(\mathbf{r}, t)$ subjected to an effective potential $v_{\text {eff }}(\mathbf{r}, t)$. This effective potential includes the external potential, the Hartree potential $v_{H}(\mathbf{r}, t)$, and an additional "exchange-correlation" (xc) potential $v_{\mathrm{xc}}(\mathbf{r}, t)$, which is a functional of the time-dependent density as well as the initial state of the system, and needs to be approximated.

The search for approximations to the $\mathrm{xc}$ potential in TDDFT has an interesting history. The first and simplest approximation, now known as the "adiabatic local density approximation" (ALDA), 5,6 actually predates the formal introduction of TDDFT. In this approximation $v_{\mathrm{xc}}(\mathbf{r}, t)$ has the same functional dependence on density as in the ground-state local density approximation, but is evaluated at the instantaneous time-dependent density. In 1985 Gross and Kohn ${ }^{7,8}$ introduced, within the framework of linear response theory, the first frequency-dependent (i.e., retarded in time) approximation to the xc potential. This approximation ${ }^{7}$ has the form

$$
v_{x c 1}(\mathbf{r}, \omega)=f_{\mathrm{xc}}(q=0, \omega) n_{1}(\mathbf{r}, \omega),
$$

where $v_{x c 1}(\mathbf{r}, \omega)$ and $n_{1}(\mathbf{r}, \omega)$ are the Fourier transforms of $v_{\mathrm{xc}}(\mathbf{r}, t)-v_{\mathrm{xc}}(\mathbf{r})$ and $n(\mathbf{r}, t)-n_{0}(\mathbf{r})$, respectively, $n_{0}(\mathbf{r})$ and $v_{\mathrm{xc}}(\mathbf{r})$ are the ground-state density and xc potential, respectively, and $f_{\mathrm{xc}}(q, \omega)$ is the so-called exchange-correlation kernel of a homogeneous electron liquid evaluated at the local ground-state density $n_{0}(\mathbf{r})$. This quantity is defined in terms of the density-density response $\chi(q, \omega)$ function as follows:

$$
\chi(q, \omega)=\frac{\chi_{0}(q, \omega)}{1-\left[v(q)+f_{\mathrm{xc}}(q, \omega)\right] \chi_{0}(q, \omega)},
$$

where $\chi_{0}(q, \omega)$ is the noninteracting density-density response function (the Lindhard function) and $v(q)$ is the Fourier transform of the Coulomb interaction.

The most important property of $f_{\mathrm{xc}}(q, \omega)$, in this context, is the existence of a finite limit for $q \rightarrow 0-$ a property that can be traced back to translational invariance, and is, of course, what makes the local density approximation (1) possible. In order to approximate $f_{\mathrm{xc}}(\omega) \equiv f_{\mathrm{xc}}(q=0, \omega)$ Gross and Kohn resorted to an interpolation formula for the spectrum $\operatorname{Im} f_{\mathrm{xc}}(\omega)$, which reduced to the exact limit of Glick and Long ${ }^{9,10} \operatorname{Im} f_{\mathrm{xc}}(\omega) \sim 1 / \omega^{3 / 2}$ at high frequency, while vanishing linearly for $\omega \rightarrow 0$. The coefficient of the linear low frequency behavior was determined by requiring the spectrum to satisfy the sum rule

$$
f_{\mathrm{xc}}(0)-f_{\mathrm{xc}}(\infty)=\int_{-\infty}^{\infty} \frac{d \omega}{\pi} \frac{\operatorname{Im} f_{\mathrm{xc}}(\omega)}{\omega},
$$

where the real quantities $f_{\mathrm{xc}}(0)$ and $f_{\mathrm{xc}}(\infty)$ were obtained from the compressibility and the third moment sum rules, respectively. ${ }^{11}$ The finite wavevector extension of the GrossKohn's approximation was later carried out by Dabrowski. ${ }^{12}$

Several aspects of this approximation are unsatisfactory. First of all, as we have already noted, the low-frequency behavior of $\operatorname{Im} f_{\mathrm{xc}}(\omega)$ is determined by global sum rule arguments, rather than being directly related to the low frequency excitation spectrum of the electron gas. The approximate formula does not take into account the possibility of specific spectral structures associated, for instance, to twoplasmon excitations. In addition, the requirement $f_{\mathrm{xc}}(0)$ $=d^{2} n \epsilon_{\mathrm{xc}}(n) / d n^{2}$ - the thermodynamic compressibility-is not quite correct: there is a subtle difference between $f_{\mathrm{xc}}(0)=\lim _{\omega \rightarrow 0} \lim _{q \rightarrow 0} f_{\mathrm{xc}}(q, \omega) \quad$ and $\quad d^{2} n \epsilon_{\mathrm{xc}}(n) / d n^{2}$ $=\lim _{q \rightarrow 0} \lim _{\omega \rightarrow 0} f_{\mathrm{xc}}(q, \omega)$. Here $\epsilon_{\mathrm{xc}}(n)$ is the exchangecorrelation energy per particle.

On a more fundamental level, it was pointed out by Dobson $^{13}$ that Eq. (1) fails to satisfy the harmonic potential theorem (intimately related to Kohn's theorem ${ }^{14}$ ). This led to a careful reconsideration of the GK dynamical LDA by sev- 
eral authors. ${ }^{13,15-17}$ The outcome of this effort was the recognition that the dynamical LDA should be formulated in terms of the current density, and that the dynamical exchange correlation potential should be replaced by an exchangecorrelation vector potential $\mathbf{A}_{\mathrm{xc}}(\mathbf{r}, \omega)$. The expression for $\mathbf{A}_{\mathrm{xc}}(\mathbf{r}, \omega)$ involves two exchange-correlation kernels $f_{\mathrm{xc}}^{L, T}(q, \omega)$ - $L "$ for "longitudinal" and " $T$ " for "transverse" - which are defined in terms of the longitudinal and transverse current-current response functions of the homogeneous electron gas as follows: ${ }^{18}$

$$
\chi^{L, T}(q, \omega)=\frac{\chi_{0}^{L, T}(q, \omega)}{1-\left(q^{2} / \omega^{2}\right)\left[v^{L, T}(q)+f_{\mathrm{xc}}^{L, T}(q, \omega)\right] \chi_{0}^{L, T}(q, \omega)},
$$

where $\chi_{0}^{L, T}(q, \omega)$ is the noninteracting response function $v^{L}(q)=v(q)$ and $v^{T}(q)=0$. It is not difficult to verify, with the help of the relation $\chi(q, \omega)=\left(q^{2} / \omega^{2}\right) \chi^{L}(q, \omega)$, that $f_{\mathrm{xc}}^{L}(q, \omega)$ coincides with the $f_{\mathrm{xc}}(q, \omega)$ of the old density functional theory.

The calculation of the longitudinal and transverse kernels $f_{\mathrm{xc}}^{L, T}(q, \omega)$ was undertaken by Nifosì, Conti, and Tosi ${ }^{19-22}$ (NCT). Unlike GK, they did not interpolate, but calculated $\operatorname{Im} f_{\mathrm{xc}}^{L, T}(\omega)$ from the approximate decoupling of an exact four-point response function. The decoupling procedure only keeps the direct contributions and ignores exchange contributions. This is equivalent to writing the spectral density of excitations, at $q=0$, as the convolution of two-single particle excitation spectra, i.e., the relevant excitations are taken to be double electron-hole pairs, double plasmons, and electron-hole-pair+plasmon excitations. A known defect of this approach is that it does not account for exchange, thus even the $\omega \rightarrow \infty$ limit is wrong. NCT corrected for this deficiency by introducing an exchange correction factor in the manner of Hubbard. This gives the correct behavior at high frequency, but is still incorrect for $\omega \rightarrow 0$. A very important feature of the NCT result in three spatial dimensions is the presence of a sharp peak in $\operatorname{Im} f_{\mathrm{xc}}^{L, T}(\omega)$ at $\omega=2 \omega_{p}$, where $\omega_{p}$ is the plasmon frequency. The occurrence of such a peak in the three-dimensional (3D) electron gas is easily understood from the mathematics of the convolution approximation: the density of single plasmon states rises sharply at the threshold at $\omega=\omega_{p}$, and so does the density of two-plasmon states at a threshold at $\omega=2 \omega_{p}$ : this leads to the rapid increase in $\operatorname{Im} f_{\mathrm{xc}}^{L, T}(\omega)$ about $2 \omega_{p}$. [In two dimensions (2D), although the plasmons still make a large contribution at some characteristic frequency, the sharp peak is absent due to the fact that the plasmon frequency vanishes at long wavelength as the square root of the wave vector.] While this physical effect is easy to understand, we believe that the simple convolution approximation probably overestimates the size of the two-plasmon peak due to lack of self-consistency. For example, it is well known that coupling of the plasmon to electron-hole pairs leads to damping of the plasmon, and consequent broadening of its spectral density of states: this should definitely broaden the sharp features found by NCT.

Another important contribution to the theory of the dynamical exchange-correlation kernel is the calculation of the frequency-dependent local field factor $G(q, i \omega)$ $=-f_{\mathrm{xc}}^{L}(q, i \omega) / v(q)$ by Richardson and Ashcroft (RA). ${ }^{23}$ This work uses a combination of perturbation theory and exact sum rules to calculate $G$ for imaginary frequencies, and has been recently shown to give excellent results for the correlation energy of the homogeneous electron gas. ${ }^{24}$ However, the limitation to imaginary frequency prevents the application of these results to truly dynamical situations.

In the present paper, we combine the interpolation philosophy of GK with the first principle approach of NCT to provide a novel and simple expression for $f_{\mathrm{xc}}^{L, T}(\omega)$ in two and three dimensions - an expression which we hope will prove superior to the ones proposed so far. Our starting point is the same as that of NCT, but, rather than relying on the decoupling approximation, we find that it is possible to calculate $\operatorname{Im} f_{\mathrm{xc}}^{L, T}(\omega)$ analytically at low frequency, i.e., we can calculate the slope of $\operatorname{Im} f_{\mathrm{xc}}^{L, T}$ versus $\omega$ at $\omega=0$ with full inclusion of the exchange terms that were ignored in this limit by NCT. In the process, we also discover an exact (i.e., nonperturbative) relation between the low-frequency limits of the longitudinal and transverse kernels $\operatorname{Im} f_{\mathrm{xc}}^{T}(q=0, \omega)$ $=[d / 2(d-1)] \operatorname{Im} f_{\mathrm{xc}}^{L}(q=0, \omega)$ at small $\omega$, where $d=3,2$ is the number of spatial dimensions.

Our calculation is done to "leading order" in the Coulomb interaction. Formally, this should be the second-order $\left[O\left(e^{4}\right)\right]$, but the long-range of the Coulomb interaction causes the second-order calculation to give a diverging result. To obtain a finite result, the interaction must be screened (by the RPA dielectric function in the weak coupling limit), but this means that higher order contributions must also be included. Of course only the leading-order term is exact: this is $O\left(e^{3}\right)$ in three dimensions and $O\left[e^{4} \ln \left(e^{2}\right)\right]$ in two dimensions.

The analytical result for the slope of $\operatorname{Im} f_{\mathrm{xc}}(\omega)$ at $\omega=0$ is then used to improve the GK's approximation. ${ }^{4,7}$ Basically we take the same approach as GK, but we include the new information on the low-frequency behavior together with the old information on the high frequency behavior and the sum rules. In order to accommodate the new constraint we need one more parameter than GK. The additional parameter is the width of a Gaussian peak centered at $\omega=2 \omega_{p}$ that we add to the original GK expression to model the two-plasmon contribution first identified by NCT. As expected, this contribution is sizeable, yet considerably smaller than in the NCT calculation.

An additional technical point that we have strived to include in our interpolation is the existence of a finite difference between $f_{\mathrm{xc}}^{L}(0)$ and the static compressibility. This difference can be expressed in terms of Landau parameters, which are known, approximately, from earlier microscopic calculations. We have taken the difference into account in calculating the parameters of our interpolation.

In summary, our expressions for $\operatorname{Im} f_{\mathrm{xc}}^{L, T}(\omega)$ and $\operatorname{Re} f_{\text {xc }}^{L, T}(\omega)$ should be more accurate, at low frequency, than either the GK or the NCT formula. In addition, the new interpolation includes a reasonable two-plasmon contribution without compromising the simplicity of the GK form. 
The paper is organized as follows. In Sec. II, we summarize the exact properties of the exchange-correlation kernels in the electron liquid. In Sec. III we present our interpolation for $f_{\mathrm{xc}}^{L, T}(\omega)$ in $3 \mathrm{D}$, and compare it with the approximations by GK and NCT. Section IV presents a similar analysis for $f_{\mathrm{xc}}^{L, T}(\omega)$ in $2 \mathrm{D}$. In Sec. V, we derive an exact relation between $\operatorname{Im} f_{\mathrm{xc}}^{L}(\omega)$ and $\operatorname{Im} f_{\mathrm{xc}}^{T}(\omega)$ at small $\omega$. The derivation of the analytical results for $\operatorname{Im} f_{\mathrm{xc}}^{L}(\omega)$ at low frequency is described in Sec. VI. Section VII contains our conclusions.

\section{EXACT PROPERTIES OF THE XC KERNELS}

The $q$-dependent kernel $f_{\mathrm{xc}}^{L, T}(q, \omega)$ is an analytic function of $\omega$ in the upper half of the complex $\omega$ plane and its real part and imaginary part satisfy Kramers-Kronig (KK) relations for each $q$. For $q=0$,

$$
\operatorname{Re} f_{\mathrm{xc}}^{L, T}(\omega)-f_{\mathrm{xc}}^{L, T}(\infty)=P \int_{-\infty}^{\infty} \frac{d \omega^{\prime}}{\pi} \frac{\operatorname{Im} f_{\mathrm{xc}}^{L, T}\left(\omega^{\prime}\right)}{\omega^{\prime}-\omega},
$$

where $P$ is the principal part. Therefore $\operatorname{Re} f_{\text {xc }}^{L, T}(\omega)$ may be obtained from the KK relation of Eq. (5) once $\operatorname{Im} f_{\mathrm{xc}}^{L, T}(\omega)$ is known.

The zero frequency limits of $f_{\mathrm{xc}}^{L}(\omega)$ and $f_{\mathrm{xc}}^{T}(\omega)$ are related to the exchange-correlation (xc) part of the elastic bulk and shear moduli $K_{\mathrm{xc}}, \mu_{\mathrm{xc}}$ via $^{26}$

$$
\begin{gathered}
f_{\mathrm{xc}}^{L}(0)=\frac{1}{n^{2}}\left[K_{\mathrm{xc}}+\frac{2(d-1)}{d} \mu_{\mathrm{xc}}\right] \equiv f_{0}^{L}(n), \\
f_{\mathrm{xc}}^{T}(0)=\frac{\mu_{\mathrm{xc}}}{n^{2}} \equiv f_{0}^{T}(n) .
\end{gathered}
$$

It is well known that

$$
K_{\mathrm{xc}}=n^{2} \frac{d^{2}}{d n^{2}}\left(n \epsilon_{\mathrm{xc}}\right),
$$

where $\epsilon_{\mathrm{xc}}(n)$ is the exchange-correlation energy per particle. The difference between Eq. (6) and the compressibility sum rule

$$
\lim _{q \rightarrow 0} \lim _{\omega \rightarrow 0} f_{\mathrm{xc}}^{L}(q, \omega)=K_{\mathrm{xc}} / n^{2}
$$

was first pointed out by Conti and Vignale. ${ }^{26}$ They further related the xc shear modulus $\mu_{\mathrm{xc}}$ to the Landau parameters $F_{l}$ as follows:

$$
\mu_{\mathrm{xc}}=\frac{2 E_{F} n}{25} \frac{3 F_{2}-5 F_{1}}{3+F_{1}}
$$

in $3 \mathrm{D}$, and

$$
\mu_{\mathrm{xc}}=\frac{E_{F} n}{2} \frac{F_{2}-F_{1}}{2+F_{1}}
$$

in $2 \mathrm{D}$, where $E_{F}=k_{F}^{2} / 2 m$.
The high frequency limits of $f_{\mathrm{xc}}^{L(T)}(\omega)$ can be expressed in terms of the ground state properties of electron gas via the third moment sum rules ${ }^{11,26}$

$$
\begin{aligned}
f_{\mathrm{xc}}^{L}(\infty)= & \frac{1}{2 n}\left[-\left(1+3 \beta^{(d)}\right) n^{1+2 / d} \frac{d}{d n}\left(\frac{\epsilon_{\mathrm{xc}}}{n^{2 / d}}\right)\right. \\
& \left.+12 n^{1 / d+1} \frac{d}{d n}\left(\frac{\epsilon_{\mathrm{xc}}}{n^{1 / d}}\right)\right] \equiv f_{\infty}^{L}(n),
\end{aligned}
$$

and

$$
\begin{aligned}
f_{\mathrm{xc}}^{T}(\infty)= & \frac{1}{2 n}\left[-\left(\beta^{(d)}-1\right) n^{1+2 / d} \frac{d}{d n}\left(\frac{\epsilon_{\mathrm{xc}}}{n^{2 / d}}\right)\right. \\
& \left.+4 n^{1 / d+1} \frac{d}{d n}\left(\frac{\epsilon_{\mathrm{xc}}}{n^{1 / d}}\right)\right] \equiv f_{\infty}^{T}(n),
\end{aligned}
$$

where $\beta^{(3)}=1 / 5$ and $\beta^{(2)}=1 / 2$.

The high-frequency behavior of $\operatorname{Im} f_{\mathrm{xc}}^{L, T}(\omega)$ is also known from second-order perturbative calculations by Glick and Long, ${ }^{9}$ Holas and Singwi, ${ }^{10}$ and $\mathrm{NCT}^{20},{ }^{20}$

$$
\operatorname{Im} f_{\mathrm{xc}}^{L, T}(\omega) \simeq-c_{d}^{L, T} \frac{\pi^{4-d} m e^{4}}{(m \omega)^{d / 2}}
$$

for $\omega \rightarrow \infty$, where $c_{3}^{L}=23 / 15, \quad c_{3}^{T}=16 / 15$ in $3 \mathrm{D}$, and $c_{2}^{L}$ $=11 / 32, c_{2}^{T}=9 / 32$ in $2 \mathrm{D}$.

In Sec. V, we prove an exact (i.e., nonperturbative) relation between the low-frequency limits of $\operatorname{Im} f_{\mathrm{xc}}^{L}(\omega)$ and $\operatorname{Im} f_{\mathrm{xc}}^{T}(\omega)$. This relation reads

$$
\lim _{\omega \rightarrow 0} \frac{\operatorname{Im} f_{\mathrm{xc}}^{T}(\omega)}{\omega}=\frac{d}{2(d-1)} \lim _{\omega \rightarrow 0} \frac{\operatorname{Im} f_{\mathrm{xc}}^{L}(\omega)}{\omega}
$$

We then show that, in Sec. VI,

$$
\lim _{\omega \rightarrow 0} \frac{\operatorname{Im} f_{\mathrm{xc}}^{L, T}(\omega)}{\omega}=-\left(\frac{m e^{2}}{n \pi}\right)^{2}\left(k_{F}\right)^{d-2} S_{d}^{L, T},
$$

where the dimensionless constant $S_{d}^{L, T}$ is given by

$$
\begin{aligned}
S_{3}^{L}= & -\frac{1}{45 \pi}\left\{5-(\lambda+5 / \lambda) \tan ^{-1} \lambda-\frac{2}{\lambda} \sin ^{-1} \frac{\lambda}{\sqrt{1+\lambda^{2}}}\right. \\
& \left.+\frac{2}{\lambda \sqrt{2+\lambda^{2}}}\left[\frac{\pi}{2}-\tan ^{-1} \frac{1}{\lambda \sqrt{2+\lambda^{2}}}\right]\right\}
\end{aligned}
$$

and

$$
S_{3}^{T}=\frac{3}{4} S_{3}^{L}
$$

in $3 \mathrm{D}$, and

$$
S_{2}^{L}=\frac{1}{6}\left\{-\frac{\pi}{4}+\frac{3-\lambda^{2}}{2-\lambda^{2}} \ln (\lambda+1)-\frac{\lambda}{1+\lambda}+\frac{1}{2-\lambda^{2}} f(\lambda)\right\}
$$


and

$$
S_{2}^{T}=S_{2}^{L}
$$

in 2D. In the above expressions $\lambda$ is defined as $\lambda$ $=2 k_{F} / k_{s}$, where $k_{s}$ is the screening wave vector $k_{s}$ $=\sqrt{4 k_{F} / \pi a_{0}}$ in $3 \mathrm{D}$ and $k_{s}=2 / a_{0}$ in $2 \mathrm{D}, k_{F}$ and $a_{0}$ are the Fermi wave vector and the Bohr radius, respectively, and

$$
f(\lambda)= \begin{cases}2 \sqrt{1-\lambda^{2}} \tan ^{-1} \sqrt{\frac{1-\lambda}{1+\lambda}}, & \text { for } \lambda<1, \\ \sqrt{\lambda^{2}-1} \ln \left[\frac{\sqrt{\lambda+1}-\sqrt{\lambda-1}}{\sqrt{\lambda+1}+\sqrt{\lambda-1}}\right], & \text { for } \lambda>1 .\end{cases}
$$

To lowest nonvanishing order in the Coulomb interaction the above results take the form

$$
\lim _{\omega \rightarrow 0} \frac{\operatorname{Im} f_{\mathrm{xc}}^{L}(\omega)}{\omega}=-\frac{1}{90}\left[\frac{k_{F}}{\pi a_{0}}\right]^{3 / 2} \frac{1}{n^{2}}
$$

in $3 \mathrm{D}$ and

$$
\lim _{\omega \rightarrow 0} \frac{\operatorname{Im} f_{\mathrm{xc}}^{L}(\omega)}{\omega}=-\frac{1}{6}\left[\frac{1}{n \pi a_{0}}\right]^{2} \ln \left(k_{F} a_{0}\right)
$$

in $2 \mathrm{D}$.

The low-frequency behavior of xc kernel has been related to the bulk and shear viscosities of the electron liquid, $\zeta$ and $\eta$ via $^{25,26}$

$$
\zeta=-n^{2} \lim _{\omega \rightarrow 0}\left[\frac{\operatorname{Im} f_{\mathrm{xc}}^{L}(\omega)}{\omega}-2 \frac{d-1}{d} \frac{\operatorname{Im} f_{\mathrm{xc}}^{T}(\omega)}{\omega}\right]
$$

and

$$
\eta=-n^{2} \lim _{\omega \rightarrow 0} \frac{\operatorname{Im} f_{\mathrm{xc}}^{T}(\omega)}{\omega}
$$

Equation (14) immediately leads to result $\zeta=0$ in both $3 \mathrm{D}$ and 2D. We note that this conclusion holds in general, i.e., beyond perturbation theory. From Eq. (15), we obtain the shear viscosity as

$$
\eta=\left[\frac{m e^{2}}{\pi}\right]^{2} k_{F}^{d-2} S_{d}^{T}
$$

This concludes our summary of the exact properties of $f_{\mathrm{xc}}^{L, T}(\omega)$.

\section{INTERPOLATION FORMULA IN THREE DIMENSIONS}

As discussed in the Introduction, our proposed interpolation for $\operatorname{Im} f_{\mathrm{xc}}^{L, T}(\omega)$ in $3 \mathrm{D}$ has the form
TABLE I. $\mu_{\mathrm{xc}}$ in $3 \mathrm{D}$ in unit $2 \omega_{\mathrm{pl}} n$.

\begin{tabular}{llllll}
\hline \hline \multicolumn{1}{c}{$r_{s}$} & \multicolumn{1}{c}{1} & \multicolumn{1}{c}{2} & \multicolumn{1}{c}{3} & \multicolumn{1}{c}{4} & \multicolumn{1}{c}{5} \\
\hline present & 0.00738 & 0.00770 & 0.00801 & 0.00837 & 0.00851 \\
NCT & 0.0064 & 0.0052 & 0.0037 & 0.0020 & 0.0002 \\
\hline \hline
\end{tabular}

$$
\begin{aligned}
\operatorname{Im} f_{\mathrm{xc}}^{L, T}(\omega)= & -\frac{2 \omega_{\mathrm{pl}}}{n} \tilde{\omega}\left[\frac{a_{3}^{L, T}}{\left(1+b_{3}^{L, T} \widetilde{\omega}^{2}\right)^{5 / 4}}\right. \\
& \left.+\tilde{\omega}^{2} e^{-\left(|\tilde{\omega}|-\Omega_{3}^{L, T}\right)^{2} / \Gamma_{3}^{L, T}}\right]
\end{aligned}
$$

where $\tilde{\omega}=\omega / \omega_{3 m}$, with $\omega_{3 m}=2 \omega_{\mathrm{pl}}$ and $\omega_{\mathrm{pl}}=\sqrt{4 \pi n e^{2} / m}$ is the plasmon frequency. We note that all the parameters $a_{3}^{L, T}$, $b_{3}^{L, T}, \Omega_{3}^{L, T}$, and $\Gamma_{3}^{L, T}$, are dimensionless. The first term in the square brackets is the Gross-Kohn interpolation formula. The second term is introduced to model the two-plasmon contribution identified in Refs. 20, 22. Requiring that this contribution be maximum near $\omega=2 \omega_{\mathrm{pl}}$ leads to a relationship between $\Omega_{3}^{L, T}$ and $\Gamma_{3}^{L, T}$

$$
\Omega_{3}^{L, T}=1-\frac{3 \Gamma_{3}^{L, T}}{2} .
$$

The low frequency result of Eq. (15) fixes $a_{3}^{L, T}$ as

$$
a_{3}^{L, T}=2\left[\frac{2}{3}\right]^{1 / 3} \pi^{-2 / 3} r_{s}^{2} S_{3}^{L, T},
$$

where $r_{s}$ is the Wigner-Seitz radius, $(4 \pi / 3) r_{s}^{3}=1 / n$. Use of the high-frequency result (13) in Eq. (26) yields

$$
b_{3}^{L, T}=16\left[\frac{2^{10}}{3 \pi^{8}}\right]^{1 / 15} r_{s}\left[\frac{S_{3}^{L, T}}{c_{3}^{L, T}}\right]^{4 / 5} .
$$

Finally, Eqs. (5) and (26) lead to the equation

$$
\begin{aligned}
& -\left(\frac{4 \sqrt{2 \pi} a_{3}^{L, T}}{[\Gamma(1 / 4)]^{2} \sqrt{b_{3}^{L, T}}}+\frac{1}{2 \pi}\left\{2 \Omega_{3}^{L, T} \Gamma_{3}^{L, T} e^{-\left(\Omega_{3}^{L, T}\right)^{2} / \Gamma_{3}^{L, T}}\right.\right. \\
& \quad+\left(\pi \Gamma_{3}^{L, T}\right)^{1 / 2}\left[\Gamma_{3}^{L, T}+2\left(\Omega_{3}^{L, T}\right)^{2}\right] \\
& \left.\left.\quad \times\left[1+\operatorname{erf}\left(\frac{\Omega_{3}^{L, T}}{\sqrt{\Gamma_{3}^{L, T}}}\right)\right]\right\}\right) \frac{2 \omega_{\mathrm{pl}}}{n} \\
& =f_{0}^{L, T}(n)-f_{\infty}^{L, T}(n),
\end{aligned}
$$

TABLE II. The parameters for $f_{\mathrm{xc}}^{L}(\omega)$ [Eq. (26)] in 3D.

\begin{tabular}{clccc}
\hline \hline$r_{s}$ & $10^{2} a_{3}^{L}$ & $b_{3}^{L}$ & $\Gamma_{3}^{L}$ & $\Omega_{3}^{L}$ \\
\hline 1 & 0.5026 & 0.1555 & 1.656 & -1.484 \\
2 & 0.8473 & 0.1558 & 1.368 & -1.052 \\
3 & 1.092 & 0.1496 & 1.215 & -0.8227 \\
4 & 1.278 & 0.1428 & 1.112 & -0.6683 \\
5 & 1.426 & 0.1363 & 1.033 & -0.5498 \\
\hline \hline
\end{tabular}


TABLE III. The parameters for $f_{\mathrm{xc}}^{T}(\omega)$ [Eq. (26)] in 3D.

\begin{tabular}{clccc}
\hline \hline$r_{s}$ & $10^{2} a_{3}^{T}$ & $b_{3}^{T}$ & $\Gamma_{3}^{T}$ & $\Omega_{3}^{T}$ \\
\hline 1 & 0.3769 & 0.1651 & 1.821 & -1.732 \\
2 & 0.6355 & 0.1654 & 1.533 & -1.300 \\
3 & 0.8191 & 0.1589 & 1.380 & -1.070 \\
4 & 0.9587 & 0.1516 & 1.277 & -0.9158 \\
5 & 1.069 & 0.1448 & 1.198 & -0.7973 \\
\hline \hline
\end{tabular}

where

$$
\operatorname{erf}(x)=\frac{2}{\sqrt{\pi}} \int_{0}^{x} e^{-y^{2}} d y
$$

By solving Eqs. (27) and (30), one determines the parameters $\Gamma_{3}^{L, T}$ and $\Omega_{3}^{L, T}$. The real parts of the xc kernels $\operatorname{Re} f_{\mathrm{xc}}^{L, T}(\omega)$ are then calculated from the KK relation of Eq. (5).

Before presenting our numerical results and comparing them with the older ones by GK (Refs. 4,7,8) and NCT, ${ }^{20-22}$ we must discuss the inputs for $f_{0}(n)$ and $f_{\infty}(n)$. For the exchange-correlation energy $\epsilon_{\mathrm{xc}}(n)$ we use the results of Monte Carlo calculations. ${ }^{27-30}$ Available results for the Landau parameter $F_{1}$, related to the electron effective mass, ${ }^{31}$ and $F_{2}$ show that $\left|\mu_{\mathrm{xc}}\right| \ll K_{\mathrm{xc}}$ at metallic densities. ${ }^{32,33}$ This is also confirmed by NCT (Ref. 22) in their RPA treatment of two-pair processes. A comparison between $\mu_{\mathrm{xc}}$ calculated by NCT and $\mu_{\mathrm{xc}}$ calculated from the Landau parameters of Ref. 33 is given in Table I. Notice that NCT put $\mu_{\mathrm{xc}}=0$ in their interpolation.

The four parameters $a, b, \Gamma$, and $\Omega$ obtained from Eqs. (27),(28), (29), and (30) are listed for several values of $r_{s}$ in Tables II and III, for $f_{\mathrm{xc}}^{L}(\omega)$ and $f_{\mathrm{xc}}^{T}(\omega)$, respectively. In Fig. 1 we plot our formula for the imaginary part of $f_{\mathrm{xc}}^{L}(\omega)$ in $3 \mathrm{D}$ at $r_{s}=3$. The GK interpolation formula ${ }^{4,7,8}$ and the result of the numerical calculation by NCT (Refs. 20,22) are also plotted for comparison. All three curves reduce to the highfrequency limit of Eq. (13). At low frequency our curve

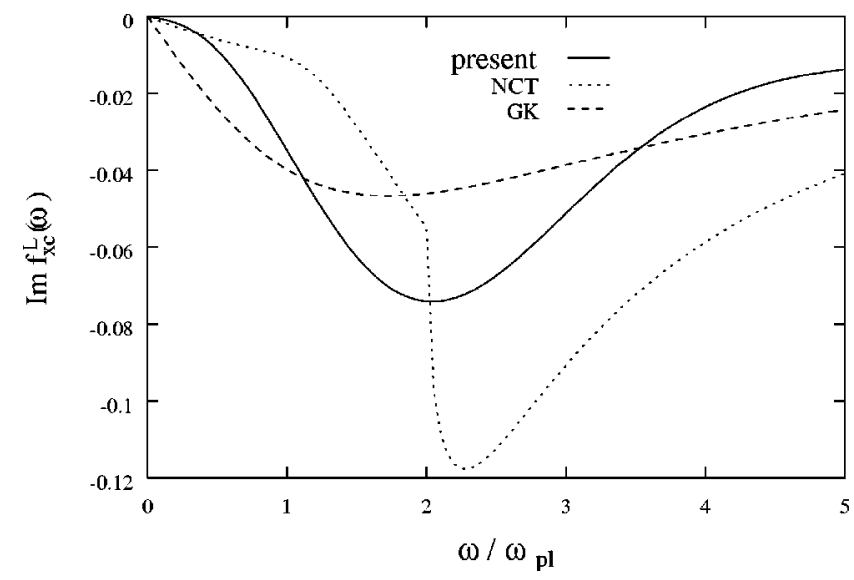

FIG. 1. Imaginary part of $f_{\mathrm{xc}}^{L}(\omega)$ in $3 \mathrm{D}$ at $r_{s}=3$ in units of $2 \omega_{\mathrm{pl}} / n$, as a function of $\omega$. Dotted line: NCT calculation; Dashed line: GK interpolation.
TABLE IV. $-\operatorname{Im} f_{\mathrm{xc}}^{L}(\omega) / \omega$ near $\omega=0$. The units are 2 $\times 10^{-2} / n$ in $3 \mathrm{D}$ and $10^{-2} / n$ in $2 \mathrm{D}$.

\begin{tabular}{ccccl}
\hline \hline$r_{s}$ & present (3D) & NCT (3D) & present (2D) & NCT (2D) \\
\hline 0.5 & 0.133 & 0.327 & 0.456 & \\
1 & 0.251 & 0.623 & 0.746 & 0.00175 \\
2 & 0.424 & 1.09 & 1.31 & 2.89 \\
3 & 0.546 & 1.42 & 1.60 & 3.49 \\
4 & 0.639 & 1.68 & 1.78 & 3.97 \\
5 & 0.713 & 1.85 & 1.91 & 4.26 \\
6 & 0.773 & 2.00 & 2.00 & 4.49 \\
7 & 0.823 & & 2.08 & \\
8 & 0.866 & & 2.14 & \\
9 & 0.903 & & 2.19 & \\
10 & 0.934 & 2.17 & 2.22 & 4.99 \\
\hline \hline
\end{tabular}

starts with a slope that is much smaller than the slope of GK, and also significantly smaller than the slope of NCT (see Table IV). This is understandable because there is no control on the value of the slope at $\omega=0$ in the GK interpolation. The difference from NCT arises from the fact that the exchange contribution cancels part of the direct contributions near $\omega=0$. The exchange correction is completely absent near $\omega=0$ in NCT's calculations because the phenomenological factor $g(\omega)$, which they introduced to simulate the contribution of the exchange, tends to one for $\omega \rightarrow 0$. Therefore, there is every reason to believe that the present results for $\operatorname{Im} f_{\mathrm{xc}}^{L}(\omega)$ at low frequency are more reliable than both GK's and NCT's.

At intermediate frequency, our results are intermediate between those of GK's and NCT's. The contribution of twoplasmon excitations at $\omega=2 \omega_{\mathrm{pl}}$ is absent in the GK interpolation, while it is quite pronounced in the present interpolation, even though the peak is not as sharp as in NCT. The real part of $f_{\mathrm{xc}}^{L}(\omega)$ is plotted in Fig. 2. Note that all the three approximations reduce to the same limits for $\operatorname{Re} f_{\mathrm{xc}}^{L}(\infty)$, as explained above.

In Figs. 3 and 4 we compare our results for $\operatorname{Im} f_{\mathrm{xc}}^{T}(\omega)$ and $\operatorname{Re} f_{\mathrm{xc}}^{T}(\omega)$ at $r_{s}=3$ with those of NCT. ${ }^{20}$ These curves are

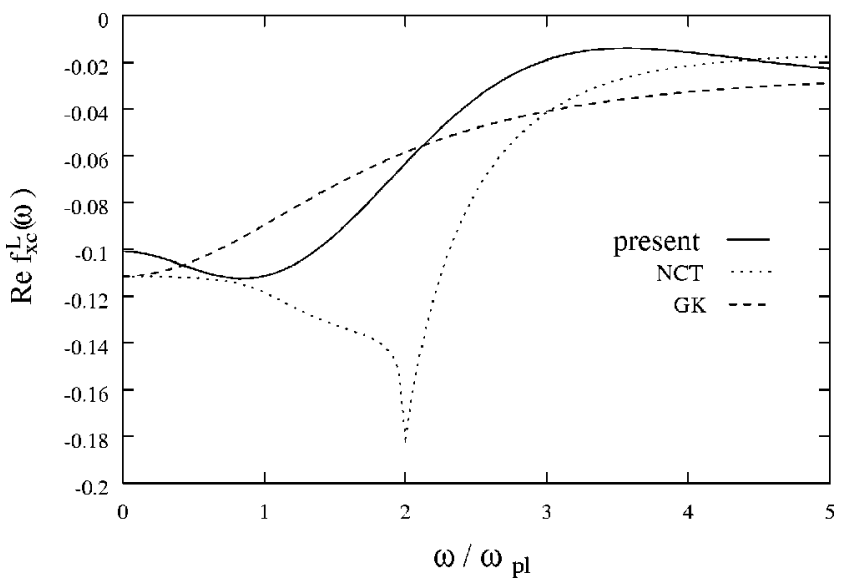

FIG. 2. Real part of $f_{\mathrm{xc}}^{L}(\omega)$ in 3D. Notations and units are as in Fig. 1. 


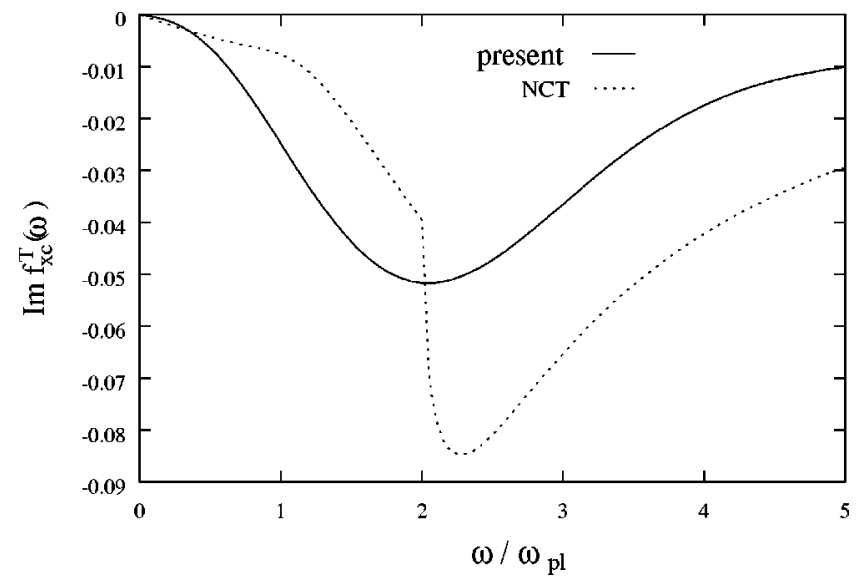

FIG. 3. Imaginary part of $f_{\mathrm{xc}}^{T}(\omega)$ in $3 \mathrm{D}$ at $r_{s}=3$ in units of $2 \omega_{\mathrm{pl}} / n$, as a function of $\omega$. Dotted line: NCT calculation.

qualitatively similar to those for $f_{\mathrm{xc}}^{L}(\omega)$. In fact $\operatorname{Im} f_{\mathrm{xc}}^{T}(\omega)$ differs from $\operatorname{Im} f_{\mathrm{xc}}^{L}(\omega)$ by an approximately constant scale factor $(\sim 0.75)$ as pointed out by NCT.

\section{INTERPOLATION FORMULA IN TWO DIMENSIONS}

The dispersion of the plasmon in a two dimensional electron liquid goes as $k^{1 / 2}$ at small wave vector $k^{10,34,35}$ This behavior is distinctly different from that of the threedimensional plasmon. Although the plasmons still make a large contribution to $\operatorname{Im} f_{\mathrm{xc}}^{L, T}(\omega)$ at a characteristic frequency $\omega_{2 m}$, a sharp threshold for two-plasmons emission is absent in 2D. Using a single mode approximation for the density fluctuation spectrum with a pole at $\omega=\sqrt{c k}+k^{2} / 2 m$ where $c=\left(r_{s} / \sqrt{2}\right)\left(2 E_{F}\right)^{2} / k_{F}$ one can estimate the characteristic two-plasmon frequency as

$$
\omega_{2 m}=\left(2^{7} r_{s}^{2}\right)^{1 / 3} E_{F}
$$

We are now in a position to approximate $\operatorname{Im} f_{\mathrm{xc}}^{L, T}(\omega)$ in $2 \mathrm{D}$ as

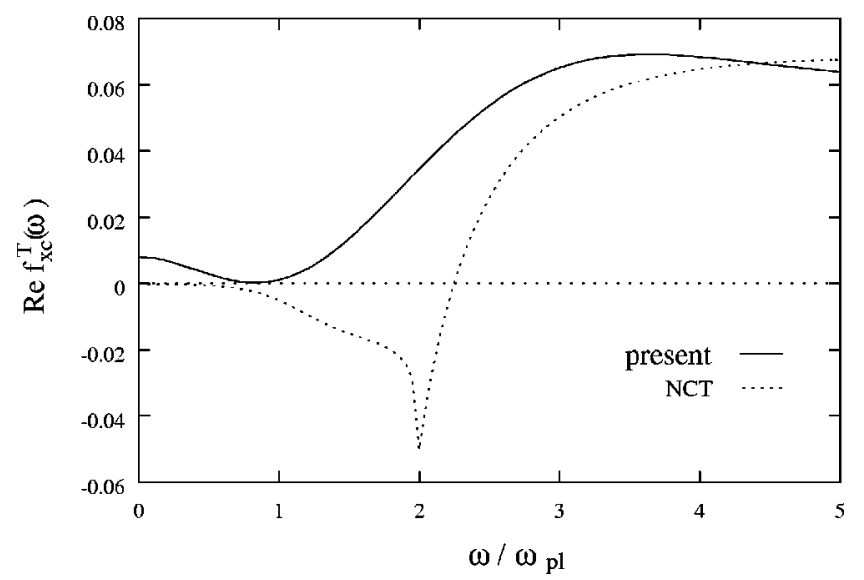

FIG. 4. Real part of $f_{\mathrm{xc}}^{T}(\omega)$ in 3D. Notations and units are as in Fig. 3.
TABLE V. The parameters for $f_{\mathrm{xc}}^{L}(\omega)$ [Eq. (33)] in 2D.

\begin{tabular}{cccccc}
\hline \hline$r_{s}$ & $10^{2} a_{2}^{L}$ & $b_{2}^{L}$ & $\Gamma_{2}^{L}$ & $\Omega_{2}^{L}$ & $10^{2} \mu_{\mathrm{xc}}(\operatorname{Ry} n)$ \\
\hline 1 & 8.448 & 0.2409 & 0.763 & -0.144 & 3.76 \\
2 & 5.260 & 0.2381 & 0.927 & -0.391 & -0.789 \\
3 & 3.716 & 0.2205 & 1.08 & -0.615 & -1.35 \\
5 & 2.250 & 0.1876 & 1.31 & -0.971 & -1.17 \\
\hline \hline & \\
& $\operatorname{Im} f_{\text {xc }}^{L, T}(\omega)=-\tilde{\omega}\left[\frac{a_{2}^{L, T}}{1+b_{2}^{L, T} \tilde{\omega}^{2}}+\tilde{\omega}^{2} e^{-\left(|\tilde{\omega}|-\Omega_{2}^{L, T}\right)^{2} / \Gamma_{2}^{L, T}}\right]\left[\frac{R y}{n}\right]$,
\end{tabular}

where $\tilde{\omega}=\omega / \omega_{2 m}$. Proceeding as in $3 \mathrm{D}$ we obtain the following four equations:

$$
\begin{gathered}
\Omega_{2}^{L, T}=1-\frac{3 \Gamma_{2}^{L, T}}{2}, \\
a_{2}^{L, T}=\pi^{-1}\left(2^{5} r_{s}\right)^{2 / 3} S_{2}^{L, T}, \\
b_{2}^{L, T}=\pi^{-2}\left(2^{7} r_{s}^{2}\right)^{2 / 3}\left[\frac{S_{2}^{L, T}}{c_{2}^{L, T}}\right],
\end{gathered}
$$

and

$$
\begin{aligned}
& -\left(\frac{a_{2}^{L, T}}{\sqrt{b_{2}^{L, T}}}+\frac{1}{2 \pi}\left\{2 \Omega_{2}^{L, T} \Gamma_{2}^{L, T} e^{-\left(\Omega_{2}^{L, T}\right)^{2} / \Gamma_{2}^{L, T}}+\sqrt{\pi \Gamma_{2}^{L, T}}\right.\right. \\
& \left.\left.\times\left[\Gamma_{2}^{L, T}+2\left(\Omega_{2}^{L, T}\right)^{2}\right]\left[1+\operatorname{erf}\left(\frac{\Omega_{2}^{L, T}}{\sqrt{\Gamma_{2}^{L, T}}}\right)\right]\right\}\right) \frac{R y}{n} \\
& =f_{0}^{L, T}(n)-f_{\infty}^{L, T}(n) .
\end{aligned}
$$

By solving Eqs. (34) and (37), we obtain the parameters $\Omega_{2}^{L, T}$ and $\Gamma_{2}^{L, T}$.

We put the screening constant $k_{s}=2 / a_{0}$ in the following numerical calculations. As in 3D, $\epsilon_{\mathrm{xc}}(n)$ is taken from Monte Carlo calculations, and the Landau parameters $F_{1}$ and $F_{2}$ are taken from the variational Monte Carlo calculations of Kwon et $\mathrm{al}^{36}$. The parameters of the interpolation are listed in Tables V and VI, and our results for $\operatorname{Im} f_{\mathrm{xc}}^{L, T}(\omega)$ at $r_{s}=3$ are plotted in Figs. 5-7, vis-a-vis the results of the NCT calculation. ${ }^{22}$ We note that the exchange-correlation contribution to the shear modulus $\mu_{\mathrm{xc}}$, calculated from the Landau parameters of Kwon et al., affects the final results for $\operatorname{Im} f_{\text {xc }}^{L, T}(\omega)$ more strongly than in three-dimensions. Aside from this difference, the qualitative behaviors of $\operatorname{Im} f_{\mathrm{xc}}^{L, T}(\omega)$

TABLE VI. The parameters for $f_{\mathrm{xc}}^{T}(\omega)$ [Eq. (33)] in 2D.

\begin{tabular}{cccccc}
\hline \hline$r_{s}$ & $10^{2} a_{2}^{T}$ & $b_{2}^{T}$ & $\Gamma_{2}^{T}$ & \multicolumn{1}{c}{$\Omega_{2}^{T}$} & $10^{2} \mu_{\text {xc }}(\operatorname{Ry} n)$ \\
\hline 1 & 8.448 & 0.1971 & 0.651 & 0.0237 & 3.76 \\
2 & 5.260 & 0.1948 & 0.861 & -0.291 & -0.789 \\
3 & 3.716 & 0.1803 & 1.03 & -0.547 & -1.35 \\
5 & 2.250 & 0.1535 & 1.29 & -0.936 & -1.17 \\
\hline \hline
\end{tabular}




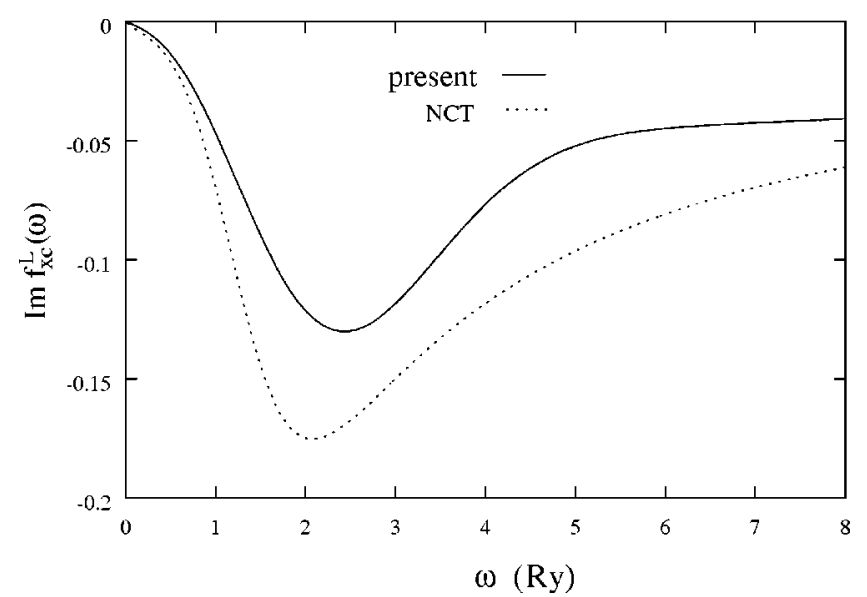

FIG. 5. Imaginary part of $f_{\mathrm{xc}}^{L}(\omega)$ in $2 \mathrm{D}$ at $r_{s}=3$ in units of $\mathrm{Ry} / n$, as a function of $\omega$. Dotted line: NCT calculation.

in $2 \mathrm{D}$ and in $3 \mathrm{D}$ are similar. As can be seen from Table III, the slopes of $f_{\mathrm{xc}}^{L}(\omega)$ near $\omega=0$ are less than half of those calculated by NCT. The real parts $f_{\mathrm{xc}}^{L, T}(\omega)$ at $r_{s}=3$ are plotted in Figs. 6 and 8, respectively. Note the reduction of the downward swing at intermediate frequencies. Our value of $\operatorname{Re} f_{\text {xc }}^{L, T}(0)$ differs from that of NCT because we make use of Monte Carlo results for $K_{\mathrm{xc}}$ and $\mu_{\mathrm{xc}}$ while they calculated these quantities from their RPA treatment of two-pair processes.

\section{EXACT RELATION BETWEEN LONGITUDINAL AND TRANSVERSE KERNELS AT LOW FREQUENCY}

In this section, we derive the exact relation (14) between $\operatorname{Im} f_{\mathrm{xc}}^{L}(\omega)$ and $\operatorname{Im} f_{\mathrm{xc}}^{T}(\omega)$ at small $\omega$. We start with the exact expression for the imaginary part of the $\mathrm{xc}$ kernel tensor

$$
f_{\mathrm{xc}, i j}(\mathbf{q}, \omega)=\frac{1}{\omega^{2}}\left[q_{i} q_{j} f_{\mathrm{xc}}^{L}(\omega)+\left(q^{2} \delta_{i j}-q_{i} q_{j}\right) f_{\mathrm{xc}}^{T}(\omega)\right],
$$

obtained by $\mathrm{NCT},{ }^{22}$ namely,

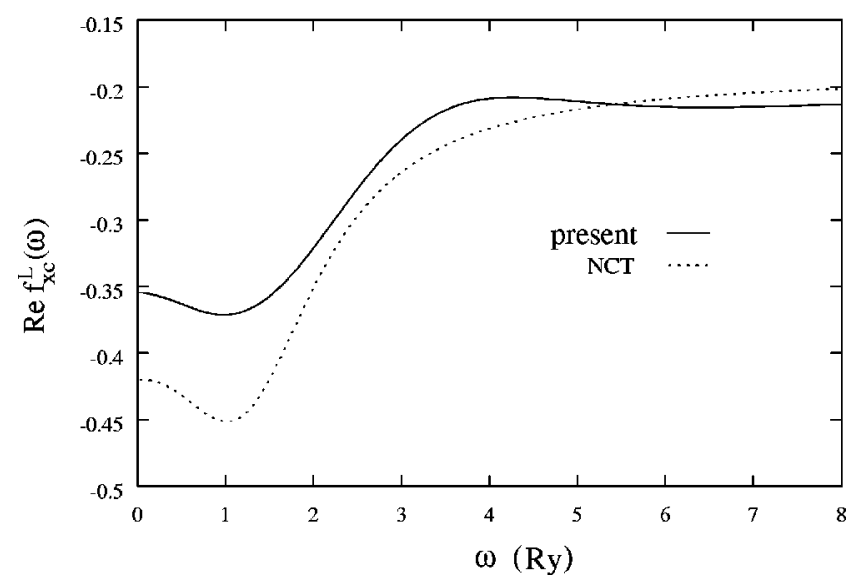

FIG. 6. Real part of $f_{\mathrm{xc}}^{L}(\omega)$ in $2 \mathrm{D}$ at $r_{s}=3$. Notations and units are as in Fig. 5.

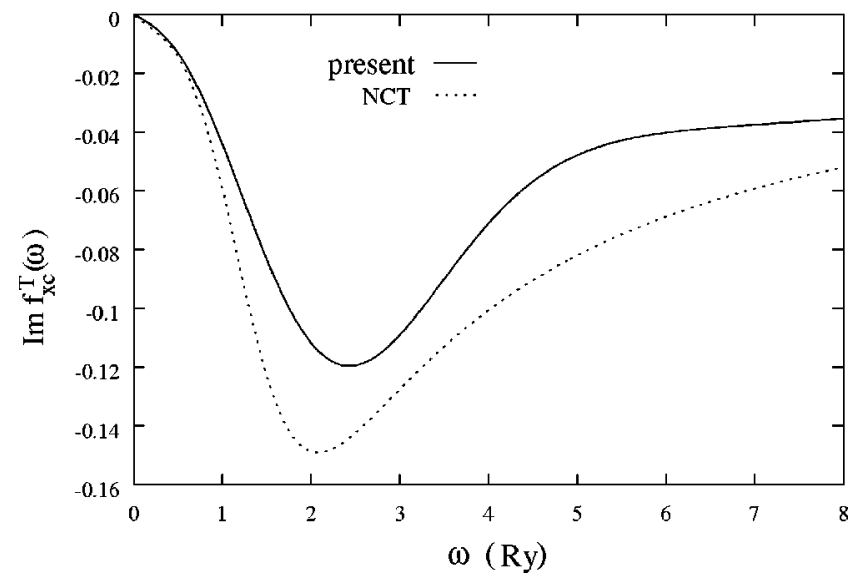

FIG. 7. Imaginary part of $f_{\mathrm{xc}}^{T}(\omega)$ in $2 \mathrm{D}$ at $r_{s}=3$ in units of $\mathrm{Ry} / n$, as a function of $\omega$. Dotted line: NCT calculation.

$$
\begin{aligned}
\operatorname{Im} f_{\mathrm{xc}, i j}(\mathbf{q}, \omega)= & \left(\frac{q}{n \omega^{2}}\right)^{2} \frac{1}{V_{d}^{3}} \lim _{q \rightarrow 0} \frac{1}{q^{2}} \sum_{\mathbf{k} \mathbf{k}^{\prime}} \sum_{l l^{\prime}} \operatorname{Im}\left\langle\left\langle j_{l}(\mathbf{k})\right.\right. \\
& \left.\left.\times \rho(-\mathbf{k}) ; j_{l^{\prime}}\left(\mathbf{k}^{\prime}\right) \rho\left(-\mathbf{k}^{\prime}\right)\right\rangle\right\rangle_{\omega} \Gamma_{i l}(\mathbf{k}, \mathbf{q}) \\
& \times \Gamma_{j l^{\prime}}\left(\mathbf{k}^{\prime},-\mathbf{q}\right),
\end{aligned}
$$

where

$$
\begin{aligned}
\Gamma_{i l}(\mathbf{k}, \mathbf{q})= & {[v(\mathbf{q}+\mathbf{k})-v(\mathbf{k})] k_{i} k_{l}+v(\mathbf{k})\left[k_{l} q_{i}-q_{l} k_{i}\right.} \\
& \left.-\delta_{i l} \mathbf{q} \cdot \mathbf{k}\right],
\end{aligned}
$$

and $i, j, l, l^{\prime}$ are Cartesian indices. Here $V_{d}$ is the volume $V$ in $3 \mathrm{D}$, the area $A$ in $2 \mathrm{D}$, and $\mathbf{j}(\mathbf{q}, t)$ is the current density operator. The Zubarev product is defined as $\langle\langle A ; B\rangle\rangle_{\omega}$ $=-i \int_{0}^{\infty} d t e^{i \omega t}\langle[A(t), B(0)]\rangle$.

For small $\omega$, all the excitations relevant to the spectrum of the four-point response function, involve states in the vicinity of the Fermi surface. Within this subset of states the longitudinal part of the current is much smaller than the transverse part, or $\hat{\mathbf{k}}[\hat{\mathbf{k}} \cdot \mathbf{j}(\mathbf{k})] \ll \mathbf{j}(\mathbf{k})$, where $\hat{\mathbf{k}}=\mathbf{k} / k$. The geometrical reason for this is shown in Fig. 9: the current operator $\mathbf{j}(\mathbf{k})=\Sigma_{\mathbf{p}}(\mathbf{p}-\mathbf{k} / 2) a_{\mathbf{p}-\mathbf{k}}^{\dagger} a_{\mathbf{p}}$ is essentially perpendicu-

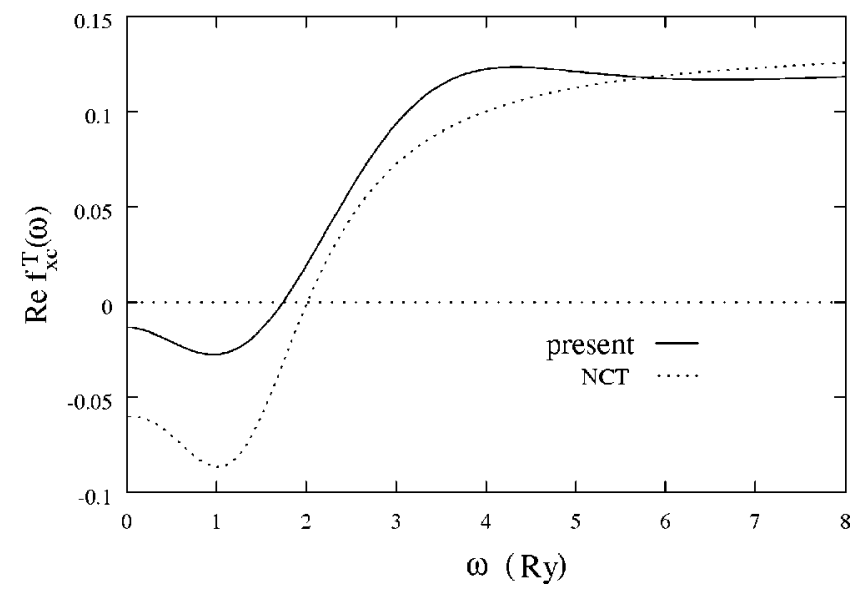

FIG. 8. Real part of $f_{\mathrm{xc}}^{T}(\omega)$ in $2 \mathrm{D}$ at $r_{s}=3$. Notations and units are as in Fig. 7. 


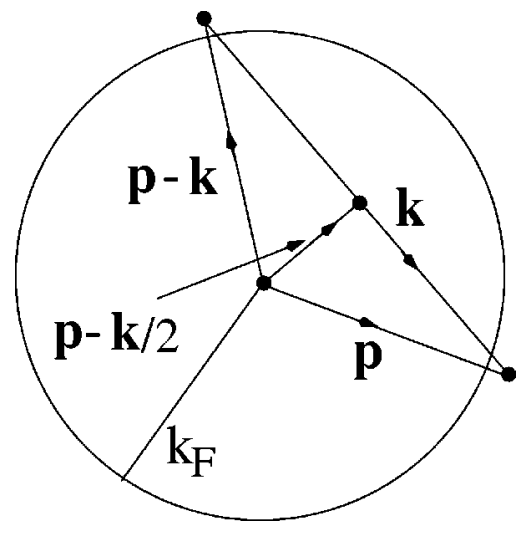

FIG. 9. Illustration of $\mathbf{k} \cdot(\mathbf{p}-\mathbf{k} / 2) \approx 0$ for low-energy electronhole excitations, which explains the suppression of the longitudinal part of the current $\mathbf{j}(\mathbf{k})$ at low frequency.

lar to $\mathbf{k}$ because $\mathbf{p}-\mathbf{k} / 2$ is essentially perpendicular to $\mathbf{k}$. Therefore, to leading order in an expansion in powers of $\omega$, $\operatorname{Im} f_{\mathrm{xc}, i j}(\mathbf{q}, \omega)$ can be simplified to

$$
\begin{aligned}
\operatorname{Im} f_{\mathrm{xc}, i j}(\mathbf{q}, \omega)= & \left(\frac{q}{n \omega^{2}}\right)^{2} \frac{1}{V_{d}^{3}} \lim _{q \rightarrow 0} \frac{1}{q^{2}} \sum_{\mathbf{k} \mathbf{k}^{\prime}} \sum_{l l^{\prime}} \operatorname{Im}\left\langle\left\langle j_{l}(\mathbf{k})\right.\right. \\
& \left.\left.\times \rho(-\mathbf{k}) ; j_{l^{\prime}}\left(\mathbf{k}^{\prime}\right) \rho\left(-\mathbf{k}^{\prime}\right)\right\rangle\right\rangle_{\omega} \\
& \times v(\mathbf{k}) v\left(\mathbf{k}^{\prime}\right) T_{i j l l^{\prime}}\left(\mathbf{k}, \mathbf{k}^{\prime}, \mathbf{q}\right),
\end{aligned}
$$

where

$$
T_{i j l l^{\prime}}\left(\mathbf{k}, \mathbf{k}^{\prime}, \mathbf{q}\right)=\left(k_{i} q_{l}+\delta_{i l} \mathbf{q} \cdot \mathbf{k}\right)\left(k_{j}^{\prime} q_{l^{\prime}}+\delta_{j l} \mathbf{k}^{\prime} \cdot \mathbf{q}\right) .
$$

We now consider the trace of the tensor $\operatorname{Im} f_{x c, i j}(\mathbf{q}, \omega)$

$$
\sum_{i j} \operatorname{Im} f_{\mathrm{xc}, i j}(\mathbf{q}, \omega) \delta_{i j}=\frac{q^{2}}{\omega^{2}}\left[\operatorname{Im} f_{\mathrm{xc}}^{L}(\omega)+(d-1) \operatorname{Im} f_{\mathrm{xc}}^{T}(\omega)\right]
$$

and its longitudinal component

$$
\sum_{i j} q_{i} q_{j} \operatorname{Im} f_{x c, i j}(\mathbf{q}, \omega)=\frac{q^{4}}{\omega^{2}} \operatorname{Im} f_{\mathrm{xc}}^{L}(\omega)
$$

The former involves the quantity

$$
\begin{aligned}
\sum_{i j} \delta_{i j} T_{i j l l^{\prime}}\left(\mathbf{k}, \mathbf{k}^{\prime}, \mathbf{q}\right)= & q_{l} q_{l^{\prime}} \mathbf{k} \cdot \mathbf{k}^{\prime}+q_{l} k_{l^{\prime}} \mathbf{k} \cdot \mathbf{q}+k_{l}^{\prime} q_{l^{\prime}} \mathbf{q} \cdot \mathbf{k} \\
& +\delta_{l l^{\prime}} \mathbf{q} \cdot \mathbf{k} \mathbf{k}^{\prime} \cdot \mathbf{q},
\end{aligned}
$$

and the latter

$$
\sum_{i j} q_{i} q_{j} T_{i j l l^{\prime}}\left(\mathbf{k}, \mathbf{k}^{\prime}, \mathbf{q}\right)=4 q_{l} q_{l^{\prime}} \mathbf{k} \cdot \mathbf{q} \mathbf{k}^{\prime} \cdot \mathbf{q} .
$$

Because the kernels $f_{\mathrm{xc}}^{L, T}(\omega)$ do not depend on the direction of $\mathbf{q}$ it is permissible to average the above expressions with respect to the direction of $\mathbf{q}$. Note that

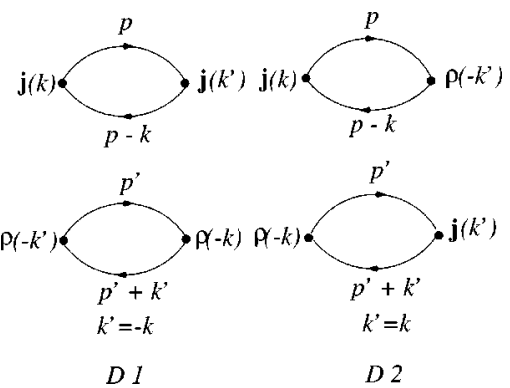

FIG. 10. The direct zeroth-order diagrams D1 and D2 of the four-point response function $\left\langle\left\langle\mathbf{j}(\mathbf{k}) \rho(-\mathbf{k}) ; \mathbf{j}\left(\mathbf{k}^{\prime}\right) \rho\left(-\mathbf{k}^{\prime}\right)\right\rangle\right\rangle_{\omega}$.

$$
\frac{1}{2^{d-1} \pi} \int d \Omega_{\mathbf{q}} \delta_{i j} T_{i j l l^{\prime}}\left(\mathbf{k}, \mathbf{k}^{\prime}, \mathbf{q}\right)=\frac{2}{d} q^{2}\left[\delta_{l l^{\prime}} \mathbf{k} \cdot \mathbf{k}^{\prime}+k_{l}^{\prime} k_{l^{\prime}}\right]
$$

and

$$
\begin{aligned}
& \frac{1}{2^{d-1} \pi} \int d \Omega_{\mathbf{q}} q_{i} q_{j} T_{i j l l^{\prime}}\left(\mathbf{k}, \mathbf{k}^{\prime}, \mathbf{q}\right) \\
& \quad=\frac{4}{d(d+2)} q^{4}\left[\delta_{l l^{\prime}} \mathbf{k} \cdot \mathbf{k}^{\prime}+k_{l} k_{l^{\prime}}^{\prime}+k_{l}^{\prime} k_{l^{\prime}}\right] .
\end{aligned}
$$

Again the term with $k_{l} k_{l}^{\prime}$ picks only the longitudinal component of the current and can therefore be dropped. Thus, to leading order in $\omega$ we get

$$
\begin{aligned}
& \int d \Omega_{\mathbf{q}} \sum_{i j} \delta_{i j} q^{2} T_{i j l l^{\prime}}\left(\mathbf{k}, \mathbf{k}^{\prime}, \mathbf{q}\right) \\
& =\frac{d+2}{2} \int d \Omega_{\mathbf{q}} \sum_{i j} q_{i} q_{j} T_{i j l l^{\prime}}\left(\mathbf{k}, \mathbf{k}^{\prime}, \mathbf{q}\right),
\end{aligned}
$$

which implies

$\sum_{i j} \delta_{i j} \operatorname{Im} f_{\mathrm{xc}, i j}(\mathbf{q}, \omega) / q^{2}=\frac{d+2}{2} \sum_{i j} \operatorname{Im} f_{x c, i j}(\mathbf{q}, \omega) q_{i} q_{j} / q^{4}$.

Together with Eqs. (43) and (44) this yields the relation (14). In the above proof, we have not made use of perturbation theory. The relation (14) is therefore exact.

\section{EVALUATION OF THE IMAGINARY PART OF THE EXCHANGE-CORRELATION KERNELS AT LOW FREQUENCY}

In this section we supply some details of the calculation of the imaginary parts of the exchange-correlation kernels at low frequency. We start from Eqs. (39) and (40). The fourpoint response function $\left\langle\left\langle j_{l}(\mathbf{k}) \rho(-\mathbf{k}) ; j_{l^{\prime}}\left(\mathbf{k}^{\prime}\right) \rho\left(-\mathbf{k}^{\prime}\right)\right\rangle\right\rangle_{\omega}$ can be expanded in a series of four-vertex diagrams such as the ones shown in Figs. 10 and 11. There are two types of diagrams. The "direct" diagrams are those that can be 


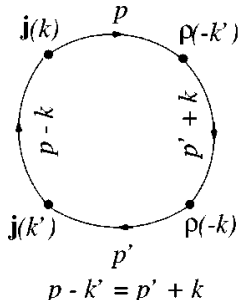

$p-k^{\prime}=p^{\prime}+k$

EXI

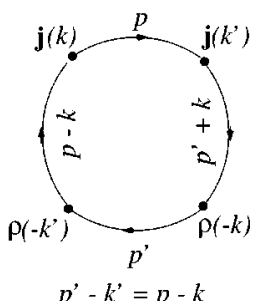

EX 2
FIG. 11. The exchange zeroth-order diagrams EX1 and EX2 of the four-point function $\left\langle\left\langle\mathbf{j}(\mathbf{k}) \rho(-\mathbf{k}) ; \mathbf{j}\left(\mathbf{k}^{\prime}\right) \rho\left(-\mathbf{k}^{\prime}\right)\right\rangle\right\rangle_{\omega}$.

separated into two parts, each containing two vertices, with no Coulomb interactions in between (see Fig. 10). The remaining diagrams are those that cannot be separated in this manner: the simplest example is the zeroth order exchange diagram shown in Fig. 11. The imaginary part of the sum of all the direct terms can be written as a convolution of the imaginary parts of exact two-point response functions as follows:

$$
\begin{aligned}
\operatorname{Im}\left\langle\left\langle j_{l}(\mathbf{k}) \rho(\mathbf{k}) ; j_{l^{\prime}}\left(\mathbf{k}^{\prime}\right) \rho\left(-\mathbf{k}^{\prime}\right)\right\rangle\right\rangle_{\omega} \\
=-\int_{0}^{\omega} \frac{d \omega^{\prime}}{\pi}\left[\operatorname{Im}\left\langle\left\langle j_{l}(\mathbf{k}) ; j_{l^{\prime}}\left(\mathbf{k}^{\prime}\right)\right\rangle\right\rangle_{\omega^{\prime}}\right. \\
\quad \times \operatorname{Im}\left\langle\left\langle\rho(\mathbf{k}) ; \rho\left(\mathbf{k}^{\prime}\right)\right\rangle\right\rangle_{\omega-\omega^{\prime}}+\operatorname{Im}\left\langle\left\langle j_{l}(\mathbf{k}) ;\right.\right. \\
\left.\left.\left.\quad \times \rho\left(-\mathbf{k}^{\prime}\right)\right\rangle\right\rangle_{\omega^{\prime}} \operatorname{Im}\left\langle\left\langle\rho(\mathbf{k}) ; j_{l^{\prime}}\left(\mathbf{k}^{\prime}\right)\right\rangle\right\rangle_{\omega-\omega^{\prime}}\right] .
\end{aligned}
$$

Using the relation

$$
\left\langle\left\langle j_{l}(\mathbf{k}) ; \rho(-\mathbf{k})\right\rangle\right\rangle=\frac{k_{l}}{k^{2}}\langle\langle\mathbf{k} \cdot \mathbf{j}(\mathbf{k}) ; \rho(-\mathbf{k})\rangle\rangle
$$

we can rewrite Eq. (51) in terms of the longitudinal and transverse linear response functions $\chi_{L}(k, \omega)$ and $\chi_{T}(k, \omega)$ as follows:

$$
\begin{aligned}
\operatorname{Im}\left\langle\left\langle j_{l}(\mathbf{k}) \rho(\mathbf{k}) ; j_{l^{\prime}}\left(\mathbf{k}^{\prime}\right) \rho\left(-\mathbf{k}^{\prime}\right)\right\rangle\right\rangle_{\omega} \\
=-V_{d}^{2} \int_{0}^{\omega} \frac{d \omega^{\prime}}{\pi} \frac{1}{k^{2}}\left\{\left[k_{l} k_{l^{\prime}} \operatorname{Im} \chi^{L}\left(\mathbf{k}, \omega^{\prime}\right)\right.\right. \\
\left.\quad+\left(k^{2} \delta_{l l^{\prime}}-k_{l^{\prime}} k_{l^{\prime}}\right) \operatorname{Im} \chi^{T}\left(\mathbf{k}, \omega^{\prime}\right)\right] \\
\quad \times \operatorname{Im} \chi^{L}\left(-\mathbf{k}, \omega-\omega^{\prime}\right) \delta_{\mathbf{k},-\mathbf{k}^{\prime}}-\frac{k_{l} k_{l^{\prime}}}{k^{2}} \omega^{\prime}\left(\omega-\omega^{\prime}\right) \\
\left.\quad \times \operatorname{Im} \chi^{L}\left(\mathbf{k}, \omega^{\prime}\right) \operatorname{Im} \chi^{L}\left(-\mathbf{k}, \omega-\omega^{\prime}\right) \delta_{\mathbf{k}, \mathbf{k}^{\prime}}\right\}
\end{aligned}
$$

Substituting Eq. (53) into Eq. (39) one obtains ${ }^{20-22}$

$$
\begin{aligned}
\operatorname{Im} f_{\mathrm{xc}}^{L, T}(\omega)= & -\int_{0}^{\omega} \frac{d \omega^{\prime}}{\pi} \int \frac{d \mathbf{k}}{(2 \pi)^{d} n^{2}}[v(k)]^{2} \\
& \times\left[\alpha_{L, T} \frac{k^{2}}{\omega^{\prime 2}} \operatorname{Im} \chi^{L}\left(k, \omega^{\prime}\right)\right. \\
& \left.+\gamma_{L, T} \frac{k^{2}}{\omega^{2}} \operatorname{Im} \chi^{T}\left(k, \omega^{\prime}\right)\right] \\
& \times \frac{k^{2}}{\left(\omega-\omega^{\prime}\right)^{2}} \operatorname{Im} \chi_{L}\left(k, \omega-\omega^{\prime}\right),
\end{aligned}
$$

where $\alpha_{L}=23 / 30$ and $\alpha_{T}=8 / 15$ in $3 \mathrm{D}$, and $\alpha_{L}=11 / 16$ and $\alpha_{T}=9 / 16$ in $2 \mathrm{D}$, while $\gamma_{L}=8 / 15$ and $\gamma_{T}=2 / 5$ in $3 \mathrm{D}$, and $\gamma_{L}=\gamma_{T}=1 / 2$ in 2D. This is the expression that NCT calculated numerically, using, for $\chi^{L, T}(k, \omega)$, the random-phase approximation (RPA) expressions

$$
\chi^{T}(k, \omega)=\chi_{0}^{T}(k, \omega)
$$

and

$$
\chi^{L}(k, \omega)=\frac{\chi_{0}^{L}(k, \omega)}{\epsilon(k, \omega)},
$$

where $\epsilon(k, \omega)=1-v(k) \chi_{0}(k, \omega)$ is the RPA dielectric function. ${ }^{37}$

The calculation simplifies considerably at low frequency, when one makes use of the limiting forms

$$
\operatorname{Im} \chi_{0}^{L}(k, \omega) \simeq 2^{5-2 d} \frac{\omega^{3}}{\pi k^{3}}\left(4 k_{F}^{2}-k^{2}\right)^{(d-3) / 2}
$$

and

$$
\operatorname{Im} \chi_{0}^{T}(k, \omega) \simeq 2^{5-3 d} \frac{\omega}{\pi k}\left(4 k_{F}^{2}-k^{2}\right)^{(d-1) / 2} .
$$

To leading order in $\omega$ we obtain

$$
\operatorname{Im} f_{\mathrm{xc}}^{L(D)}(\omega)=-\frac{m^{2} \omega}{720 \pi^{5} n^{2}} \int_{0}^{2 k_{F}} \frac{[v(k)]^{2}}{|\epsilon(k, 0)|^{2}}\left(4 k_{F}^{2}-k^{2}\right) k^{2} d k
$$

in 3D (the superscript " $D$ " stands for "direct") and

$$
\operatorname{Im} f_{\mathrm{xc}}^{L(D)}(\omega)=-\frac{m^{2} \omega}{24 \pi^{4} n^{2}} \int_{0}^{2 k_{F}} k \frac{[v(k)]^{2}}{|\epsilon(k, 0)|^{2}} d k,
$$

in 2D. In writing these expressions we have used the fact that the dynamical dielectric function $\epsilon(k, \omega)$ can be replaced by the static dielectric function $\epsilon(k, 0)$ to leading order in $\omega$. Notice that, due to the long range of the Coulomb interaction, the integrals in Eqs. (59), (60) would diverge if we used the zeroth order approximation $\epsilon(k, 0)=1$. Use of the 
RPA static dielectric function assures that we include all the contributions of leading order in the strength of the Coulomb interaction.

Carrying out the integrals over $k$ in Eqs. (59), (60) we get

$\operatorname{Im} f_{\mathrm{xc}}^{L(D)}(\omega)=-\frac{\omega e^{4} m^{2} k_{F}}{45 \pi^{3} n^{2}}\left[-3+(\lambda+3 / \lambda) \tan ^{-1} \lambda\right]$

in $3 \mathrm{D}$, and

$$
\operatorname{Im} f_{\mathrm{xc}}^{L(D)}(\omega)=-\frac{m^{2} \omega e^{4}}{6 \pi^{2} n^{2}}\left[\ln (\lambda+1)-\frac{\lambda}{\lambda+1}\right]
$$

in 2D. The dimensionless constant is defined as $\lambda$ $=2 k_{F} / k_{s}$. Notice that Eq. (59) corrects the result previously reported by Conti and Vignale in Ref. 26, whereas Eq. (60) agrees with Eq. (4.6) of Ref. 26 for $d=2$.

We now come to the calculation of the exchange contributions - the terms that are missing in the NCT calculation for low frequency. To leading order in the Coulomb interaction only the two diagrams shown in Fig. 11 need to be considered. After some calculations, we get

$$
\begin{aligned}
\operatorname{Im}\left\langle\left\langle j_{l}(\mathbf{k}) \rho(-\mathbf{k}) ; j_{l^{\prime}}\left(\mathbf{k}^{\prime}\right) \rho\left(-\mathbf{k}^{\prime}\right)\right\rangle\right\rangle_{\omega}^{(\mathrm{EX})} \\
=\frac{2 \pi}{m^{2}} \sum_{\mathbf{p} \mathbf{p}^{\prime}}(\mathbf{p}-\mathbf{k} / 2)_{l}\left\{\left(\mathbf{p}-\mathbf{k}-\mathbf{k}^{\prime} / 2\right)_{l^{\prime}} \delta_{\mathbf{p}^{\prime}+\mathbf{k}^{\prime}, \mathbf{p}-\mathbf{k}}\right. \\
\left.\quad+\left(\mathbf{p}^{\prime}+\mathbf{k}-\mathbf{k}^{\prime} / 2\right)_{l^{\prime}} \delta_{\mathbf{p}+\mathbf{k}^{\prime}, \mathbf{p}^{\prime}+\mathbf{k}}\right\} \bar{n}_{\mathbf{p}} n_{\mathbf{p}-\mathbf{k}} \bar{n}_{\mathbf{p}^{\prime}} n_{\mathbf{p}^{\prime}+\mathbf{k}} \\
\quad \times \delta\left(\omega+\omega_{-\mathbf{k p}}+\omega_{\mathbf{k} \mathbf{p}^{\prime}}\right),
\end{aligned}
$$

where $n_{\mathbf{p}}=\theta\left(k_{F}-p\right), \quad \bar{n}_{\mathbf{p}}=1-n_{\mathbf{p}}$, and $\omega_{\mathbf{k p}} \equiv \omega_{\mathbf{k}+\mathbf{p}}-\omega_{\mathbf{p}}$. The contribution from the first term in the above equation corresponds to "EX1" and the second one to "EX2" of Fig. 11. They can be evaluated in the same way. In fact, it can be readily shown that they make equivalent contributions to $\operatorname{Im} f_{\mathrm{xc}}(\omega)$ at small $\omega$. In other words, to first order in $\omega$

$$
\operatorname{Im} f_{\mathrm{xc}}^{L(\mathrm{EX})}(\omega)=2 \operatorname{Im} f_{\mathrm{xc}}^{L(\mathrm{EX} 1)}(\omega) .
$$

Therefore we focus on the first term only, which is given by

$$
\begin{aligned}
\operatorname{Im}\left\langle\left\langle j_{l}(\mathbf{k}) \rho(-\mathbf{k}) ; j_{l^{\prime}}\left(\mathbf{k}^{\prime}\right) \rho\left(-\mathbf{k}^{\prime}\right)\right\rangle\right\rangle_{\omega}(\mathrm{EX} 1) \\
=\frac{2 \pi}{m^{2}} \sum_{\mathbf{p}}(\mathbf{p}-\mathbf{k} / 2)_{l}\left(\mathbf{p}-\mathbf{k}-\mathbf{k}^{\prime} / 2\right)_{l^{\prime}} \bar{n}_{\mathbf{p}} n_{\mathbf{p}-\mathbf{k}^{n}} \bar{n}_{\mathbf{p}-\mathbf{k}-\mathbf{k}^{\prime}} \\
\quad \times n_{\mathbf{p}-\mathbf{k}^{\prime}} \delta\left(\omega+\omega_{-\mathbf{k p}}-\omega_{-\mathbf{k p}-\mathbf{k}^{\prime}}\right) .
\end{aligned}
$$

The evaluation of this complex expression is made relatively easier by the use of the following relation:

$$
\begin{aligned}
\bar{n}_{\mathbf{p}} n_{\mathbf{p}-\mathbf{k}} & \bar{n}_{\mathbf{p}-\mathbf{k}-\mathbf{k}^{\prime}} n_{\mathbf{p}-\mathbf{k}^{\prime}} \delta\left(\omega+\omega_{-\mathbf{k p}}-\omega_{-\mathbf{k p}-\mathbf{k}^{\prime}}\right) \\
= & \bar{n}_{\mathbf{p}} n_{\mathbf{p}-\mathbf{k}} \bar{n}_{\mathbf{p}-\mathbf{k}-\mathbf{k}^{\prime}} n_{\mathbf{p}-\mathbf{k}^{\prime}} \int_{0}^{\omega} d \omega^{\prime} \delta\left(\omega^{\prime}-\omega_{-\mathbf{k p}-\mathbf{k}^{\prime}}\right) \\
& \times \delta\left(\omega-\omega^{\prime}+\omega_{-\mathbf{k p}}\right)
\end{aligned}
$$

for $\omega>0$. Note that $\operatorname{Im} f_{\mathrm{xc}}^{L}(\omega)$ is an odd function of $\omega$ and therefore it is sufficient to consider only the $\omega>0$ case. The contribution of Eq. (65) to $\operatorname{Im} f_{\mathrm{xc}}^{L(\mathrm{EX} 1)}(\omega)$ can be expressed as

$$
\operatorname{Im} f_{\mathrm{xc}}^{L(\mathrm{EX} 1)}(\omega)=2 \pi\left(\frac{1}{m n \omega}\right)^{2} \frac{1}{V_{d}^{3}} Q(\omega)
$$

where

$$
\begin{aligned}
Q(\omega)= & \lim _{q \rightarrow 0} \frac{1}{q^{4}} \sum_{\mathbf{p}} \sum_{\mathbf{k}} \sum_{\mathbf{k}^{\prime}} \int_{0}^{\omega} d \omega^{\prime} \bar{n}_{\mathbf{p}} n_{\mathbf{p}-\mathbf{k}} \bar{n}_{\mathbf{p}-\mathbf{k}-\mathbf{k}^{\prime}} n_{\mathbf{p}-\mathbf{k}^{\prime}} \\
& \times \delta\left(\omega^{\prime}-\omega_{-\mathbf{k p}-\mathbf{k}^{\prime}}\right) \delta\left(\omega-\omega^{\prime}+\omega_{-\mathbf{k p}}\right) \Lambda\left(\mathbf{p}, \mathbf{k}, \mathbf{k}^{\prime} ; \mathbf{q}\right) .
\end{aligned}
$$

$\Lambda\left(\mathbf{p}, \mathbf{k}, \mathbf{k}^{\prime} ; \mathbf{q}\right)$ in Eq. (68) is defined as

$$
\begin{aligned}
\Lambda\left(\mathbf{p}, \mathbf{k}, \mathbf{k}^{\prime} ; \mathbf{q}\right)= & \sum_{l l^{\prime}}(\mathbf{p}-\mathbf{k} / 2){ }_{l}\left(\mathbf{p}-\mathbf{k}-\mathbf{k}^{\prime} / 2\right)_{l^{\prime}} \Gamma_{l}(\mathbf{k}, \mathbf{q}) \\
& \times \Gamma_{l^{\prime}}\left(\mathbf{k}^{\prime},-\mathbf{q}\right),
\end{aligned}
$$

where

$$
\Gamma_{l}(\mathbf{k}, \mathbf{q})=[v(\mathbf{q}+\mathbf{k})-v(\mathbf{k})] \mathbf{q} \cdot \mathbf{k} k_{l}+v(\mathbf{k})\left[q^{2} k_{l}-2 \mathbf{k} \cdot \mathbf{q} q_{l}\right] .
$$

The leading term of $Q(\omega)$ is proportional to $\omega^{3}$. The fact that $Q$ is independent of $q$ allows us to rewrite Eq. (68) as

$$
\begin{aligned}
Q(\omega)= & \sum_{\mathbf{p}} \sum_{\mathbf{k}} \sum_{\mathbf{k}^{\prime}} \int_{0}^{\omega} d \omega^{\prime} \bar{n}_{\mathbf{p}} n_{\mathbf{p}-\mathbf{k}} \bar{n}_{\mathbf{p}-\mathbf{k}-\mathbf{k}^{\prime}} n_{\mathbf{p}-\mathbf{k}^{\prime}} \\
& \times \delta\left(\omega^{\prime}-\omega_{-\mathbf{k p}-\mathbf{k}^{\prime}}\right) \delta\left(\omega-\omega^{\prime}+\omega_{-\mathbf{k p}}\right) \\
& \times \lim _{q \rightarrow 0} \frac{1}{q^{4}} 2^{1-d} \pi^{-1} \int d \Omega_{\mathbf{q}} \Lambda\left(\mathbf{p}, \mathbf{k}, \mathbf{k}^{\prime} ; \mathbf{q}\right) .
\end{aligned}
$$

The integral in Eq. (71) can be done with the help of formulas such as

$$
\begin{aligned}
\int & d \Omega_{\mathbf{q}}\left(\mathbf{M}_{0} \cdot \hat{\mathbf{q}}\right)^{2}\left(\mathbf{M}_{1} \cdot \hat{\mathbf{q}}\right)\left(\mathbf{M}_{2} \cdot \hat{\mathbf{q}}\right) \\
= & \frac{2^{d-1} \pi}{d(d+2)}\left[2\left(\mathbf{M}_{1} \cdot \mathbf{M}_{0}\right)\left(\mathbf{M}_{2} \cdot \mathbf{M}_{0}\right)+M_{0}^{2} \mathbf{M}_{1} \cdot \mathbf{M}_{2}\right],
\end{aligned}
$$

for arbitrary vectors $\mathbf{M}_{0}, \mathbf{M}_{1}, \mathbf{M}_{2}$. After a tedious but straightforward algebra, we obtain

$$
\begin{aligned}
Q(\omega)= & \frac{2}{d(d+2)} \int_{0}^{\omega} d \omega^{\prime} \sum_{\mathbf{k}} \sum_{\mathbf{p}} v(\mathbf{k}) k^{2} \bar{n}_{\mathbf{p}} n_{\mathbf{p}-\mathbf{k}} \\
& \times \delta\left(\omega-\omega^{\prime}+\omega_{-\mathbf{k p}}\right) J\left(\omega^{\prime}, \mathbf{k}, \mathbf{p}\right)
\end{aligned}
$$

where

$$
J\left(\omega^{\prime}, \mathbf{k}, \mathbf{p}\right)=\sum_{\mathbf{k}^{\prime}} v\left(\mathbf{k}^{\prime}+\mathbf{p}\right) \mathbf{p} \cdot\left(\mathbf{k}^{\prime}+\mathbf{p}\right) \bar{n}_{\mathbf{k}+\mathbf{k}^{\prime}} n_{\mathbf{k}^{\prime}} \delta\left(\omega^{\prime}-\omega_{\mathbf{k k}^{\prime}}\right)
$$

In reaching Eq. (73), we made use of the fact that $\mathbf{k} \cdot \mathbf{k}^{\prime}$ $=m \omega$ and $\mathbf{k} \cdot(\mathbf{p}-\mathbf{k} / 2)=m\left(\omega-\omega^{\prime}\right)$, from the $\delta$ functions in Eq. (71), and dropped all terms higher than the leading order 
in $\omega$. In fact, all these higher order terms are related to the longitudinal component of the current. Therefore the details of the derivation of Eq. (73), which are not presented here, can be greatly simplified by using from the very beginning the fact that the longitudinal component of the currents is suppressed at small $\omega$, as pointed out in the preceding section. In the following subsections, we evaluate $Q(\omega)$ in 3D and 2D separately.

\section{A. 3D}

To calculate $J\left(\omega^{\prime}, \mathbf{k}, \mathbf{p}\right)$ in $3 \mathrm{D}$ we use the statically screened Coulomb potential $v(k)=4 \pi e^{2} /\left(k^{2}+k_{s}^{2}\right)$ rather than the bare one $\left(4 \pi e^{2} / k^{2}\right)$. Although this is clearly an uncontrolled approximation beyond second order in the Coulomb interaction, it at least assures that we are calculating the true exchange counterpart of the direct terms computed in the previous section (also uncontrolled beyond the second order).

To linear order in $\omega^{\prime}, J\left(\omega^{\prime}, \mathbf{k}, \mathbf{p}\right)$ in 3D can be evaluated as

$$
J\left(\omega^{\prime}, \mathbf{k}, \mathbf{p}\right)=\theta\left(2 k_{F}-k\right) \frac{m^{2} \omega^{\prime} e^{2} V}{2 \pi k}[1+G(\mathbf{p}, \mathbf{k})],
$$

where

$$
\begin{aligned}
G(\mathbf{p}, \mathbf{k})= & {\left[p^{2}-k_{s}^{2}-k_{F}^{2}\right]\left\{\left[k_{F}^{2}+k_{s}^{2}+p^{2}-\mathbf{p} \cdot \mathbf{k}\right]^{2}\right.} \\
& \left.-\left[p^{2}-(\mathbf{p} \cdot \hat{\mathbf{k}})^{2}\right]\left[4 k_{F}^{2}-k^{2}\right]\right\}^{-1 / 2} .
\end{aligned}
$$

Equations (73), (75), and (76) yield

$$
\begin{aligned}
Q(\omega)= & \frac{m^{2} e^{2} V}{15 \pi} \int_{0}^{\omega} \omega^{\prime} d \omega^{\prime} \sum_{\mathbf{k}} v(\mathbf{k}) k \theta\left(2 k_{F}-k\right) \\
& \times\left[\frac{m^{2}\left(\omega-\omega^{\prime}\right) V}{(2 \pi)^{2} k}+I_{3}\right],
\end{aligned}
$$

where

$$
I_{3}=\sum_{\mathbf{p}} \bar{n}_{\mathbf{p}} n_{\mathbf{p}-\mathbf{k}} \delta\left(\omega-\omega^{\prime}+\omega_{-\mathbf{k p}}\right) G(\mathbf{p}, \mathbf{k}) .
$$

The linear order term of Eq. (78) can be readily established as

$$
I_{3}=-\frac{m^{2} V\left(\omega-\omega^{\prime}\right)}{(2 \pi)^{2} k} \frac{k_{s}}{\sqrt{k_{s}^{2}+4 k_{F}^{2}-k^{2}}} .
$$

Putting Eqs. (79) into (77), we obtain the low-frequency expression for $Q(\omega)$ as

$$
\begin{aligned}
Q(\omega)= & \left(\frac{m}{2 \pi}\right)^{4} \frac{2 \pi e^{2} V^{2} \omega^{3}}{45} \sum_{\mathbf{k}} v(\mathbf{k}) \theta\left(2 k_{F}-k\right) \\
& \times\left[1-\frac{k_{s}}{\sqrt{k_{s}^{2}+4 k_{F}^{2}-k^{2}}}\right] .
\end{aligned}
$$

Carrying out the integral in Eq. (80), and substituting the result into Eq. (67) we finally obtain

$$
\begin{aligned}
\operatorname{Im} f_{\mathrm{xc}}^{L(\mathrm{EX})}(\omega)= & \frac{2 \omega m^{2} e^{4} k_{F}}{45 \pi^{3} n^{2}}\left\{1-\frac{1}{\lambda} \sin ^{-1} \frac{\lambda}{\sqrt{1+\lambda^{2}}}\right. \\
& -\frac{1}{\lambda} \tan ^{-1} \lambda+\frac{1}{\lambda \sqrt{2+\lambda^{2}}} \\
& \left.\times\left[\frac{\pi}{2}-\tan ^{-1} \frac{1}{\lambda \sqrt{2+\lambda^{2}}}\right]\right\} .
\end{aligned}
$$

[We have also used Eq. (64)]. Adding $\operatorname{Im} f_{\mathrm{xc}}^{L(\mathrm{EX})}(\omega)$ of Eq. (81) to $\operatorname{Im} f_{\mathrm{xc}}^{L(D)}(\omega)$ of Eq. (61) leads to the result for $\operatorname{Im} f_{\mathrm{xc}}^{L}(\omega)$ in $3 \mathrm{D}$ in Eq. (15).

\section{B. 2D}

In $2 \mathrm{D}$, making use of the statically screened Coulomb potential $v(k)=2 \pi e^{2} /\left(k+k_{s}\right)$ we obtain

$$
J\left(\omega^{\prime}, \mathbf{k}, \mathbf{p}\right)=\theta\left(2 k_{F}-k\right) \frac{m^{2} e^{2} A \omega^{\prime}}{\pi k \sqrt{4 k_{F}^{2}-k^{2}}} H(\mathbf{p}, \mathbf{k}),
$$

where

$$
\begin{aligned}
H(\mathbf{p}, \mathbf{k})= & \frac{1}{2}\left\{\frac{2 p^{2}-\mathbf{p} \cdot \mathbf{k}+D(\mathbf{p}, \mathbf{k})}{\sqrt{\mathbf{p} \cdot(\mathbf{p}-\mathbf{k})+k_{F}^{2}+D(\mathbf{p}, \mathbf{k})}+k_{s}}\right. \\
& \left.+\frac{2 p^{2}-\mathbf{p} \cdot \mathbf{k}-D(\mathbf{p}, \mathbf{k})}{\sqrt{\mathbf{p} \cdot(\mathbf{p}-\mathbf{k})+k_{F}^{2}-D(\mathbf{p}, \mathbf{k})}+k_{s}}\right\},
\end{aligned}
$$

with $D(\mathbf{p}, \mathbf{k})=p \sqrt{4 k_{F}^{2}-k^{2}} \sin \theta$, and $\cos \theta=\hat{\mathbf{p}} \cdot \hat{\mathbf{k}}$. Equations (73) and (82) yield

$$
Q(\omega)=\frac{m^{2} e^{2} A}{4 \pi} \int_{0}^{\omega} \omega^{\prime} d \omega^{\prime} \sum_{\mathbf{k}} v(\mathbf{k}) \frac{k}{\sqrt{4 k_{F}^{2}-k^{2}}} \theta\left(2 k_{F}-k\right) I_{2},
$$

where

$$
I_{2}=\sum_{\mathbf{p}} \bar{n}_{\mathbf{p}} n_{\mathbf{p}-\mathbf{k}} \delta\left(\omega-\omega^{\prime}+\omega_{-\mathbf{k p}}\right) H(\mathbf{p}, \mathbf{k}) .
$$

The linear order term of Eq. (85) can be evaluated as

$$
I_{2}=\frac{m^{2}\left(\omega-\omega^{\prime}\right) A}{2 \pi^{2} k}\left[1-\frac{k_{s}}{\sqrt{4 k_{F}^{2}-k^{2}}+k_{s}}\right] .
$$

$Q(\omega)$ at low frequency is then obtained as

$$
Q(\omega)=\left(\frac{m}{2 \pi}\right)^{4} \frac{\pi e^{2} A^{2} \omega^{3}}{3} \sum_{\mathbf{k}} \frac{v(k)}{\sqrt{4 k_{F}^{2}-k^{2}}+k_{s}} \theta\left(2 k_{F}-k\right) .
$$

Finally, we have

$\operatorname{Im} f_{\mathrm{xc}}^{L(\mathrm{EX})}(\omega)=\frac{m^{2} e^{4} \omega}{12 \pi^{2} n^{2}} \int_{0}^{1} d x \frac{\lambda^{2} x}{\lambda x+1} \frac{1}{\left(\lambda \sqrt{1-x^{2}}+1\right)}$. 
We have used Eq. (64) in obtaining Eq. (88). The integral in the above equation may be evaluated analytically as

$$
\operatorname{Im} f_{\mathrm{xc}}^{L(\mathrm{EX})}(\omega)=\frac{m^{2} e^{4} \omega}{12 \pi^{2} n^{2}}\left\{\frac{\pi}{2}-\frac{2}{2-\lambda^{2}}[\ln (1+\lambda)+f(\lambda)]\right\},
$$

where the function $f(\lambda)$ is defined in Eq. (20). Equations (62) and (89) lead to the result for $\operatorname{Im} f_{\mathrm{xc}}^{L}(\omega)$ in $2 \mathrm{D}$ in Eq. (15).

\section{SUMMARY}

In this paper we have introduced a new analytical approximation for the longitudinal and transverse exchange and correlation kernels in the three and two-dimensional electron liquid. The new expressions represent an improvement upon the Gross-Kohn formulas because the imaginary part of the $f_{\mathrm{xc}}(\omega)$ kernels is now exact at low frequency to lowest nonvanishing order in the strength of the Coulomb interaction. This was accomplished through an exact evaluation of the leading exchange diagrams in the diagrammatic expansion of the imaginary part of $f_{\mathrm{xc}}(\omega)$. Thus, we have also improved upon the recent NCT calculation, in which exchange effects were approximated through an empirical correction factor. The additional exact constraint at low frequency cannot be satisfied by the Gross-Kohn formula, if one insists on satisfying the other known sum rules. Thus, we modified the GK formula by adding a new term which produces a gaussian peak at about two times the plasmon frequency in 3D (or the equivalent characteristic frequency in 2D). The existence of such a peak is suggested by the NCT calculation, but we believe that the strength of the peak is probably overestimated in that calculation. Our results are intermediate between the GK formula, which does not take into account the physical effect of two-plasmon emission, and the NCT result.

We have also examined the delicate question of the $\omega \rightarrow 0$ limit of $f_{\text {xc }}^{L}(\omega)$, which we now know does not coincide with the compressibility. We estimate the difference between the exact limit and the compressibility and find that it is rather small at densities of interest in $3 \mathrm{D}$, but in $2 \mathrm{D}$, this correction is more significant.

Finally we note that the analytical formula of Eq. (15) is easy to use in practical calculations. This makes the application of the present work to the low frequency dynamics particularly promising.

\section{ACKNOWLEDGMENTS}

We gratefully acknowledge support for this work from the NSF Grant No. DMR-0074959 and from the Research Board Grant No. URB-00-029 at the University of Missouri.
${ }^{1}$ P. Hohenberg and W. Kohn, Phys. Rev. 136, B864 (1964).

${ }^{2}$ W. Kohn and L. J. Sham, Phys. Rev. 140, A1133 (1965).

${ }^{3}$ E. Runge and E. K. U. Gross, Phys. Rev. Lett. 77, 2037 (1984).

${ }^{4}$ E. K. U. Gross, J. F. Dobson, and M. Petersilka, in Topics in Current Chemistry, edited by R. F. Nalewajski (Springer, Berlin, 1996).

${ }^{5}$ A. Zangwill and P. Soven, Phys. Rev. A 21, 1561 (1980).

${ }^{6}$ A. Zangwill and P. Soven, Phys. Rev. Lett. 45, 204 (1980).

${ }^{7}$ E. K. U. Gross and W. Kohn, Phys. Rev. Lett. 55, 2850 (1985); 57, 923(E) (1986).

${ }^{8}$ N. Iwamoto and E. K. U. Gross, Phys. Rev. B 35, 3003 (1987).

${ }^{9}$ A. J. Glick and W. F. Long, Phys. Rev. B 4, 3455 (1971).

${ }^{10}$ A. Holas and K. Singwi, Phys. Rev. B 40, 158 (1989).

${ }^{11}$ S. Ichimaru, Rev. Mod. Phys. 54, 1017 (1982).

${ }^{12}$ B. Dabrowski, Phys. Rev. B 34, 4989 (1986).

${ }^{13}$ J. Dobson, Phys. Rev. Lett. 73, 2244 (1994).

${ }^{14}$ W. Kohn, Phys. Rev. 123, 1242 (1961).

${ }^{15}$ G. Vignale, Phys. Rev. Lett. 74, 3233 (1995).

${ }^{16}$ G. Vignale and W. Kohn, Phys. Rev. Lett. 77, 2037 (1996).

${ }^{17}$ G. Vignale and W. Kohn, in Electronic Density Functional Theory, edited by J. Dobson, M. P. Das, and G. Vignale (Plenum Press, New York, 1996).

${ }^{18}$ K. S. Singwi and M. P. Tosi, in Solid State Physics, edited by H. Ehrenreich, F. Seitz, and D. Turnbull (Academic, New York, 1981), Vol. 36, p. 177.

${ }^{19}$ H. M. Bohm, S. Conti, and M. P. Tosi, J. Phys.: Condens. Matter 8, 781 (1996).
${ }^{20}$ S. Conti, R. Nifosì, and M. P. Tosi, J. Phys.: Condens. Matter 9, L475 (1997).

${ }^{21}$ R. Nifosi, S. Conti, and M. P. Tosi, Physica E (Amsterdam) 1, 188 (1997)

${ }^{22}$ R. Nifosi, S. Conti, and M. P. Tosi, Phys. Rev. B 58, 12758 (1998).

${ }^{23}$ C. F. Richardson and N. W. Ashcroft, Phys. Rev. B 50, 8170 (1994).

${ }^{24}$ M. Lein, E. K. U. Gross, and J. J. Perdew, Phys. Rev. B 61, 13431 (2000).

${ }^{25}$ G. Vignale, C. A. Ullrich, and S. Conti, Phys. Rev. Lett. 79, 4878 (1997).

${ }^{26}$ S. Conti and G. Vignale, Phys. Rev. B 60, 7966 (1999).

${ }^{27}$ S. H. Vosko, L. Wilk, and M. Nusair, Can. J. Phys. 58, 1200 (1980).

${ }^{28}$ F. Rapisarda and G. Senatore, Aust. J. Phys. 49, 161 (1996).

${ }^{29}$ D. M. Ceperley, Phys. Rev. B 18, 3126 (1978).

${ }^{30}$ D. M. Ceperley and B. J. Alder, Phys. Rev. Lett. 45, 566 (1980).

${ }^{31}$ For example, L. Hedin, Phys. Rev. 139, A796 (1965); T. M. Rice, Ann. Phys. (N.Y.) 31, 100 (1965).

${ }^{32}$ T. M. Rice, Phys. Rev. 175, 858 (1968).

${ }^{33}$ H. Yasuhara and Y. Ousaka, Int. J. Mod. Phys. B 6, 3089 (1992).

${ }^{34}$ F. Stern, Phys. Rev. Lett. 18, 546 (1967).

${ }^{35}$ A. Czachor, A. Holas, S. R. Sharma, and K. S. Singwi, Phys. Rev. B 25, 2144 (1982).

${ }^{36}$ Y. Kwon, D. M. Ceperley, and R. M. Martin, Phys. Rev. B 50, 1684 (1994).

${ }^{37}$ The longitudinal part of Eq. (54) in $d=3$ was first derived by M. Hasegawa and W. Watabe, J. Phys. Soc. Jpn. 27, 1393 (1969). 